\title{
Phasic norepinephrine is a neural interrupt signal for unexpected events in rapidly unfolding sensory sequences - evidence from pupillometry
}

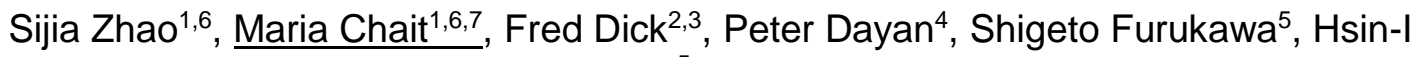 \\ Liao $^{5}$ \\ ${ }^{1}$ Ear Institute, University College London, London WC1X 8EE, UK \\ 2 Department of Psychological Sciences, Birkbeck College, London, WC1E 7HX \\ ${ }^{3}$ Department of Experimental Psychology, University College London, WC1H ODS \\ ${ }^{4}$ Max Planck Institute for Biological Cybernetics, 72076 Tübingen, Germany \\ ${ }^{5}$ NTT Communication Science Laboratories, NTT Corporation, Atsugi, 243-0198 Japan \\ ${ }^{6}$ Corresponding authors \\ ${ }^{7}$ Lead contact
}

Lead Contact:

Maria Chait

m.chait@ucl.ac.uk

Ear Institute, University College London

332 Gray's Inn Road, London WC1X 8EE, UK

Corresponding Authors:

Maria Chait

m.chait@ucl.ac.uk

Ear Institute, University College London

332 Gray's Inn Road, London WC1X 8EE, UK

\section{Sijia Zhao}

sijia.zhao.10@ucl.ac.uk

Ear Institute, University College London

332 Gray's Inn Road, London WC1X 8EE, UK

Number of figures: 7

Number of pages: 42 (51 with figures embedded)

Keywords: Noradrenaline; Unexpected Uncertainty; surprise; prediction error; Human; Eye tracking 


\section{Summary}

The ability to track the statistics of our surroundings is a key computational challenge. A prominent theory (Dayan \& Yu, 2006) proposes that the brain monitors for 'unexpected uncertainty' - events which deviate substantially from model predictions, indicating model failure. Norepinephrine (NE) is thought to play a key role in this process by serving as an interrupt signal, initiating model-resetting. However, evidence is from paradigms where participants actively monitored stimulus statistics. To determine whether NE routinely reports the statistical structure of our surroundings, even when not behaviourally relevant, we used rapid tone-pip sequences that contained perceptually salient pattern-changes associated with abrupt structural violations vs. emergence of regular structure. Phasic pupil dilations (PDR) were monitored to assess NE. We reveal a remarkable specificity: When not behaviourally relevant, only abrupt structural violations evoked a PDR. The results demonstrate that NE tracks 'unexpected uncertainty' on rapid time scales relevant to sensory signals. 


\section{Introduction}

A growing body of work demonstrates that observers maintain detailed models of the statistics of their environments over various timescales, combining this information with sensory input to inform choice (Rushworth and Behrens, 2008), increase response accuracy (Krishnamurthy et al., 2017), speed up reaction times (Bestmann et al., 2008; Marshall et al., 2016), and improve detection (Sohoglu and Chait, 2016; Southwell and Chait, 2018). A key challenge in this context is keeping track of the evolving input statistics so as to ensure model validity. Here we investigated automatic and controlled aspects of the neural response to this challenge in a fast-paced domain.

For effective model maintenance, a central dilemma faced by the brain is to arbitrate between gradual and punctate changes in the environment (Gershman et al., 2013). In the former case, model-updating progresses at a steady pace, dictated by the model's estimate of local noise ('expected uncertainty') arising from tracked environmental stochasticities (Bland and Schaefer, 2012; O’Reilly, 2013). However, environments can also change substantially and suddenly. The ability to detect such change points is crucial for optimal behavior, because they indicate that the observer's beliefs about the environment are no longer a valid representation of reality, and should be reset (Marshall et al., 2016; Payzan-LeNestour et al., 2013). For example, in Nassar et al. (2012) subjects were instructed to predict sequentiallypresented numbers drawn from a Gaussian distribution whose mean occasionally changed abruptly. Following change points, participants tended to alter their behavior in a way that reflected abandonment of old expectations and more rapid acquisition of new ones - e.g., recent events had more influence on decisions than those occurring further in the past, equivalent to an increased learning (and forgetting) rate. 
Although such change processes can often be described optimally in hierarchical probabilistic terms, an alternative heuristic is for the brain to monitor events that fall outside the threshold of 'expected uncertainty' estimated for the model, and treat them as signaling potential change points in the environment. Such so-called 'unexpected uncertainty' (Dayan and $\mathrm{Yu}, 2006)$ has been suggested as interrupting top-down processes so as to prioritize bottom-up evidence accumulation, thereby speeding up discovery of the new structure of the environment (Dayan and Yu, 2006; O’Reilly, 2013; Sara and Bouret, 2012). The neuromodulator norepinephrine (alternatively noradrenaline or NE) has been hypothesized to play a critical role in this updating process (Bouret and Sara, 2005; Dayan and Yu, 2006; Marshall et al., 2016; Payzan-LeNestour et al., 2013; Yu and Dayan, 2005). NE is generated in the brainstem nucleus Locus Coeruleus (LC), which projects extensively across the brain and spinal cord (Samuels and Szabadi, 2008a, 2008b; Sara and Bouret, 2012) and is thus optimally placed to signal a global state change in the environment. However, a vast literature has also implicated NE in controlling vigilance, orienting behavior, selective attention and surprise (Aston-Jones and Cohen, 2005; Avery and Krichmar, 2017; Sara and Bouret, 2012), suggesting that it might instead play a much less specific role, associated with regulating arousal.

The bulk of work on NE and model updating in humans has involved paradigms in which participants actively monitor the statistics of the stimulus, for instance through explicit tracking tasks (Krishnamurthy et al., 2017; Nassar et al., 2012; Payzan-LeNestour et al., 2013; Preuschoff et al., 2011) or in speeded stimulus-response paradigms (Marshall et al., 2016). It is therefore an open question whether NE involvement is driven by behavioral relevance (for decisions or motor responses), or if NE plays a more ubiquitous role in reporting changes in the statistical structure of our surroundings. In the latter case, we need to examine which events trigger its release. 
Sensory systems continuously analyze probabilistic information which unfolds on a rapid timescale, even when this information is not immediately relevant to behavior (Barascud et al., 2016; Sohoglu and Chait, 2016; Turk-Browne et al., 2010). It is therefore compelling to ask (1) how the fast-paced and automatic mechanisms that detect changes in statistics within rapid sensory signals interface with NE, (2) how NE's involvement compares with other aspects of neural dynamics, and (3) what effect there is, if any, of making the changes behaviorally consequential. In addition, by understanding the contingencies to which NE responds, we hope to gain extra clarity on the heuristic separation between gradual and punctate change that is critical for effective model maintenance.

To examine these questions, we sought a sensory paradigm that induces such changes, along with a way of assessing the effect on NE and other neural systems. For the first, we considered rapid auditory patterns (Fig. 1A) consisting of sequences of tone-pips (new on each trial) containing transitions either from a repeating or 'regular' (REG) to a random (RAND) frequency structure, or the reverse. These stimuli are particularly suited for our purposes since at a presentation rate of $20 \mathrm{~Hz}$, the sequences are too rapid for naive listeners to explicitly follow the unfolding pattern. Rather, the changes in sequence structure (in both directions) readily 'pop out' from the stimulus stream irrespective of subjective effort (see stimulus examples in sup. materials or https://goo.gl/vddYuS). Furthermore, the changes induce patterns of neural dynamics (Barascud et al., 2016; Southwell et al., 2017) which hint that they might illuminate the central dilemma about punctate versus gradual change. Transitions from regular to random frequency structures evoke a prompt mismatch neural response, triggered by the abrupt violation of the regular pattern. The opposite transitions, random to regular - despite having matched overall spectro-temporal structure and being similarly detectable - do not generate a mismatch response. Instead, the dynamics of the brain response are consistent with an evidence accumulation process which changes more slowly from one structure to the other. 
In order to assess NE, we turned to the eyes. Indirect measures of NE release can be obtained from monitoring non-luminance-mediated changes in pupil size (Aston-Jones and Cohen, 2005; Joshi et al., 2016). This renders pupillometry an attractive, non-invasive means of probing NE activity in the brain. There is a consistent mechanistic correlation between spiking activity in the LC and changes in pupil size, both when spontaneously occurring, and when triggered by external events (Joshi et al., 2016; Reimer et al., 2016). In particular, transient pupil dilation responses (PDR) have been shown to causally relate to phasic activity within the LC-NE system (Joshi et al., 2016; Reimer et al., 2016), though there remains uncertainty about the specific circuitry (Costa and Rudebeck, 2016). Capitalizing on these links, recent pupillometry studies have revealed a relationship between pupil dilation and predictability (Krishnamurthy et al., 2017; Nassar et al., 2012), providing (indirect) evidence for the involvement of phasic LC-NE responses in signaling uncertainty.

Thus, we monitored pupil size whilst subjects listened to changing auditory sequences, including the disambiguating regular-random (REG-RAND) and random-regular (RAND-REG) transition types. If the pupil-linked LC-NE system generally monitors for salient state changes in the environment, both transition types are expected to evoke PDRs. However, under the hypothesis that phasic LC-NE responses are selective for punctate changes even when detected automatically in speeded inputs, we should observe pupil dilation responses to the former, but not the latter, transition. By also asking participants explicitly to monitor for and report both types of transitions, we could see if the coupling to NE responses is obligatory or behaviorally penetrable - i.e., whether the range of statistical model violations that determine NE activity can be influenced by instructions. 


\section{Results}

The basic stimulus set is shown in Fig. $1 \mathrm{~A}$. In referring to the stimuli we adopt a nomenclature where the term in uppercase denotes the type of signal (RAND vs REG) and the subscript indicates to the size of the sub-pool from which the relevant pattern is created. Thus, $\mathrm{RAND}_{20}$ is a tone series created by randomly selecting each tone (with replacement) from a full pool of 20 frequencies. RAND 10 is a series created from a subset of 10 different frequencies (randomly selected from the full pool), while $R E G_{10}$ is a regular pattern consisting of a repeating sequence of 10 tones (a different pattern, and a different sub-pool on each trial).

Brain response (MEG and EEG) data suggest that while $R E G_{10}-R A N D_{20}$ and $R A N D_{20}-$ $R E G_{10}$ transitions are characterized by opposite statistics (emergence vs. violation of regularity), both are detected automatically, and at a similar latency even when participants' attention is directed elsewhere (Barascud et al., 2016; Southwell et al., 2017). When asked to respond behaviorally to transitions, listeners exhibit ceiling performance and similar reaction times with comparable variability (Barascud et al. 2016, also replicated here in Exp. 3). Thus, these signals are well suited for disambiguating the role of the pupil linked LC-NE system in tracking statistics of rapidly-evolving sensory signals.

To control for overall engagement (Jepma and Nieuwenhuis, 2011) and ensure broad attention to the auditory stimuli, but without requiring active tracking of the transitions, naïve participants (in Exp. 1,2,4) detected short silent gaps as they listened to the tone-pip sequences. Gap occurrence was uncorrelated to state transition, and the subset of sequences containing gaps were excluded from analyses. (In Exp. 3 we investigate the effect of making the state transition task-relevant). 
A.
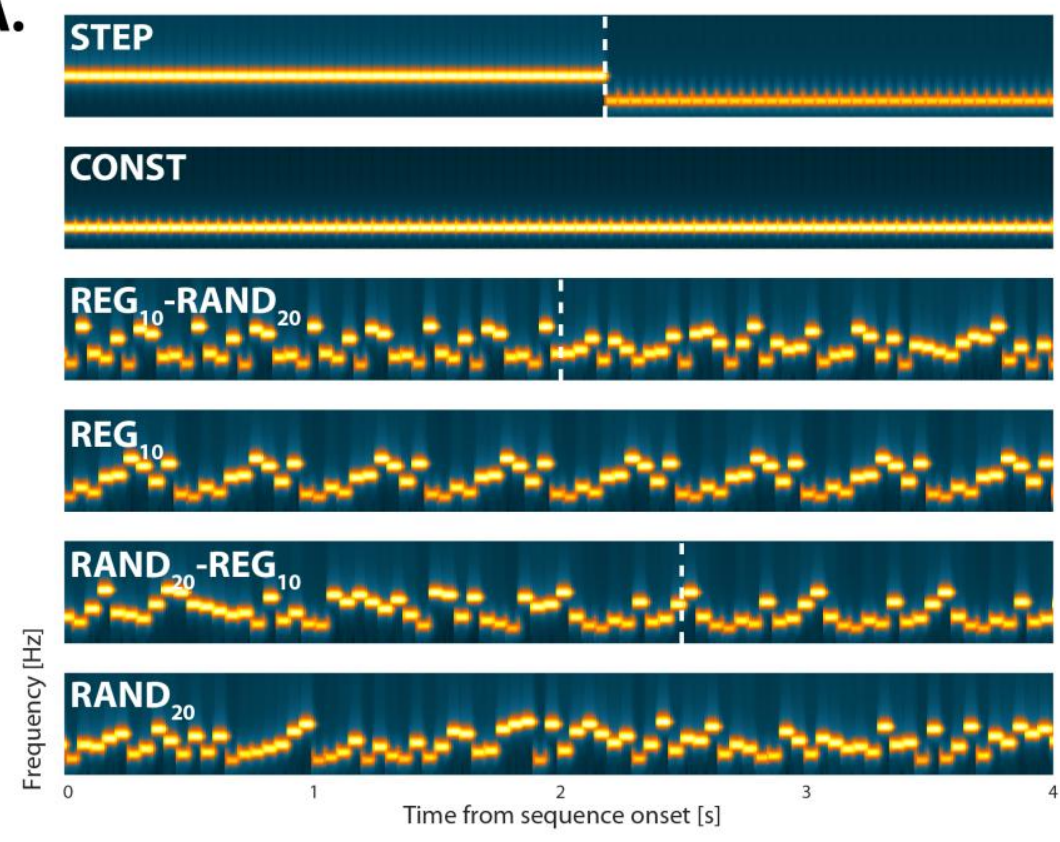

B. MEG results from Barascud et al. (2016)

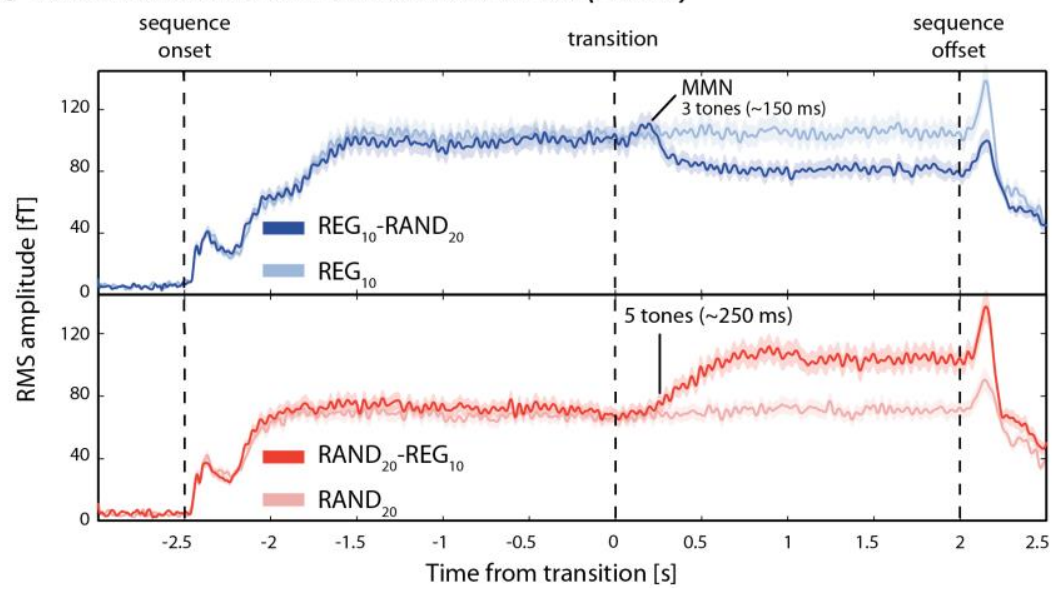

Figure 1: Example spectrograms of basic stimuli, and brain responses to REG-RAND and RAND-REG transitions recorded with MEG. [A] The stimuli were sequences of concatenated tone-pips (50ms) with frequencies drawn from a pool of 20 fixed values. The tone-pips were arranged according to 6 frequency patterns, generated anew for each subject and on each trial: CONST sequences consisted of a single repeating tone; STEP contained a step change from one tone frequency to another; $R E G_{10}$ sequences were generated by randomly selecting 10 frequencies from the pool and iterating that sequence to create a regularly repeating pattern; $R A N D_{20}$ were generated by randomly sampling from the full pool with replacement; $R E G_{10}-R_{A N D_{20}}$ and $R A N D_{20}-R G_{10}$ sequences contained a transition between a regular and random pattern or vice versa. Transition times (between 2.5 - 3.5s post onset) are indicated by a white dashed line. In RAND $_{20}-\mathrm{REG}_{10}$ sequences, the transition time is defined as occurring after the first full regularity cycle, i.e. once the transition becomes statistically detectable. For presentation purposes only, the plotted sequence lengths are equal. Durations varied randomly between 5 to $7 \mathrm{~s}$. [B] Brain responses $(\mathrm{N}=13)$ to $R E G_{10}-\mathrm{RAND}_{20}$ (top panel) and RAND $_{20}-$ REG $_{10}$ (bottom panel), together with their no-change controls, recorded with 
magnetoencephalography (MEG). Plotted is Root Mean Square (RMS) over channels, as an estimate of instantaneous power. The figures show the entire stimulus epoch, relative to the transition. Shaded areas are \pm 1 SEM. The transition from RAND to REG is associated with a gradual increase in sustained power from $\sim 250 \mathrm{~ms}$ (5 tones) post transition. The transition from REG to RAND evokes an MMN-like response (at $\sim 150 \mathrm{~ms}$ after the transition) followed by a sharp drop in the sustained response. These changes in power are hypothesized to reflect the instantiation (RAND-REG) or interruption (REGRAND) of a contextual top-down model. See Barascud et al. (2016) for more details. 


\section{Exp. 1: The pupil dilates to violation but not emergence of regularity in complex tone patterns}

\section{$\operatorname{Exp} .1 A(N=18)$}

Fig. 2A plots the average normalized pupil size data across all participants as a function of time relative to the transition. Clear PDRs were observed in the STEP and REG ${ }_{10}-R_{A N D_{20}}$ conditions, but not in the $R A N D_{20}-R E G_{10}$ condition.

Performance on the gap detection task was good overall (Fig. 2E) but we observed a main effect of condition on hit rates (arc-sine transformed for these and all subsequent statistical analyses on hit rates, $F(1.198,20.372)=8.285, p=0.007)$. Post hoc tests confirmed that hit rate in $\operatorname{RAND}_{20}$ was lower than in CONST $(p=0.026)$ and $\mathrm{REG}_{10}(p=0.026)$, while CONST and $R E G_{10}$ did not differ significantly $(p=1.000)$. To assure that performance disparities were not driving differential PDR effects, gap duration in Exp. 1B was lengthened by $50 \mathrm{~ms}$ to equate task performance across conditions.

\section{$\operatorname{Exp} 1 B(N=14)$}

The revised paradigm successfully eliminated performance differences between conditions (Fig. 2F), with a repeated-measures ANOVA showing no effect of stimulus condition on hits $(F(2,26)=2.115, p=0.141)$ or false positive rates $(F(2,26)=1.000, p=1.000)$.

The PDR pattern observed in Exp. 1A was entirely replicated (Fig. 2B). Overall, the results of Exp. 1 confirmed that PDRs are consistently evoked by STEP and REG ${ }_{10}-R_{A N D_{20}}$ transitions, but not $\mathrm{RAND}_{20}-\mathrm{REG}_{10}$ transitions. 


\section{Experiment $1 \mathrm{~A}$}

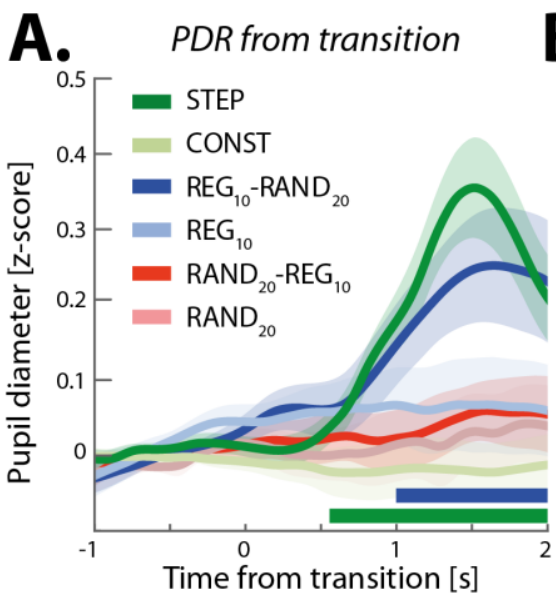

\section{Experiment 1B}

B.

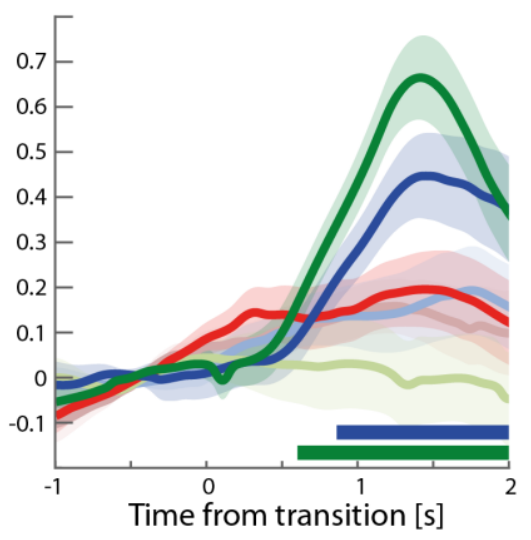

PDR from onset

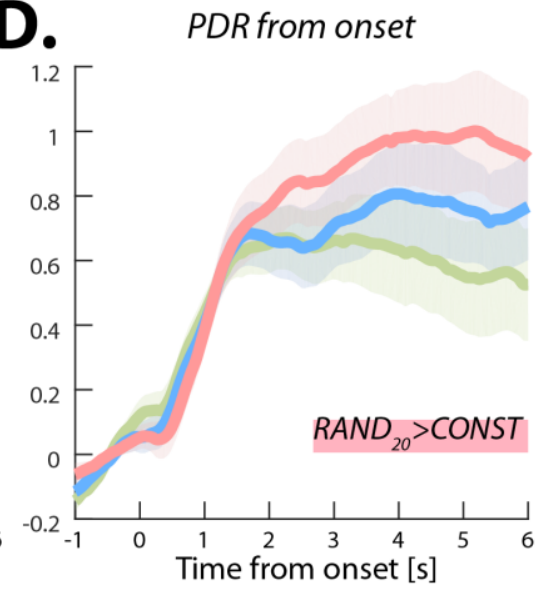

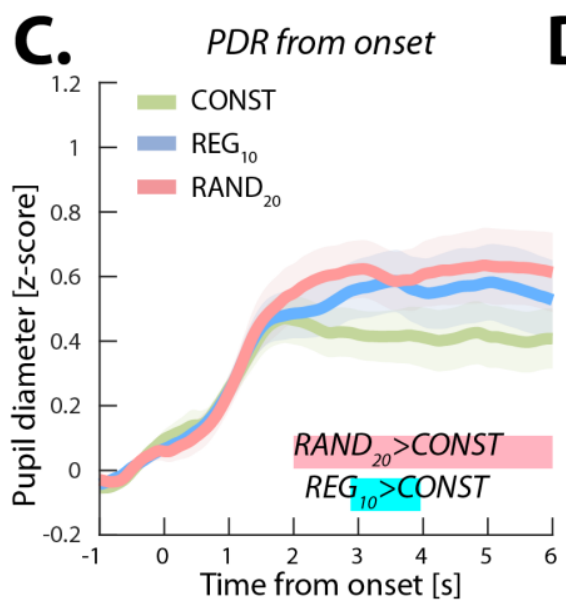

F.

E. Behavioural performance
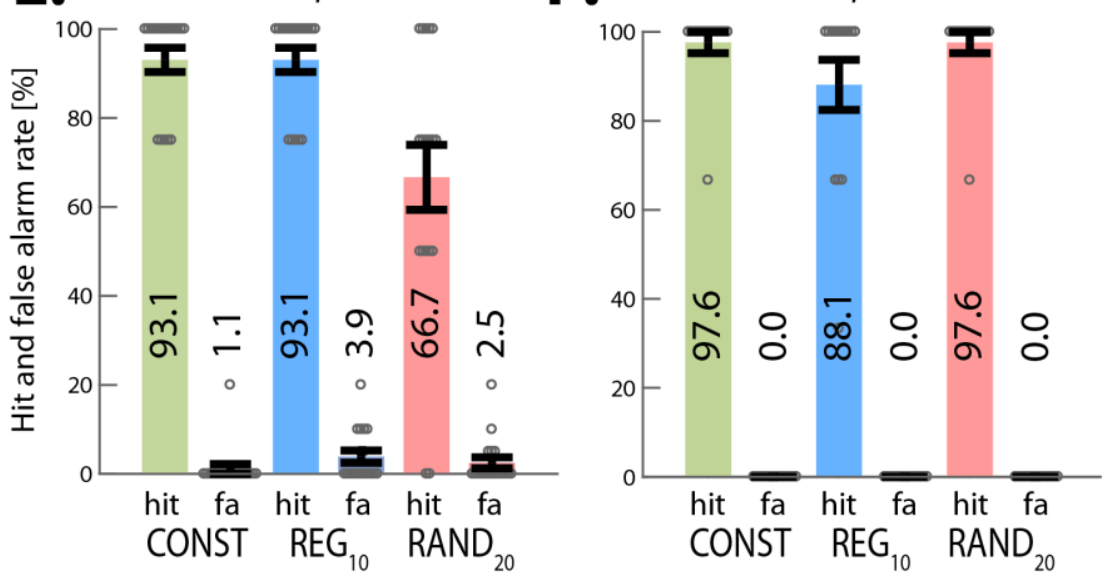

Figure 2: Experiment 1 (1A: $\mathrm{N}=18,1 \mathrm{~B}: \mathrm{N}=14$ ). REG-RAND but not RAND-REG transitions are associated with a pupil dilation response. [A] Average pupil diameter over time relative to the transition in Experiment $1 \mathrm{~A}$. Solid lines represent the average normalized pupil diameter. The shaded area shows \pm 1 SEM. Color-coded horizontal lines at graph bottom indicate time intervals where cluster-level statistics show significant differences between each change condition and its no-change control $(p<0.05)$. In STEP, the pupil diameter started to increase around $300 \mathrm{~ms}$ post-transition, reaching peak amplitude at 
$1520 \mathrm{~ms}$; it statistically diverged from its control, CONST, from 560ms through to sequence offset. Similarly, the PDR to REG ${ }_{10}-$ RAND $_{20}$ increased from $\sim 700 \mathrm{~ms}$ posttransition, peaking at $1640 \mathrm{~ms}$. $R E G_{10}-R{ }^{2} D_{20}$ statistically diverged from its control, $R_{E G_{10}}$, at $1000 \mathrm{~ms}$ post-transition and remained significantly higher until $2400 \mathrm{~ms}$. No significant differences between $R A N D_{20}-R E G_{10}$ and $R A N D_{20}$ were observed. Transition conditions were also compared directly (not shown): $\mathbf{R E G}_{10}-\mathbf{R A N D}_{20}$ was significantly higher than $\mathrm{RAND}_{20}-\mathrm{REG}_{10}$ from $840 \mathrm{~ms}$ post-transition and up to stimulus offset, but no difference was observed between STEP and $R E G_{10}-R_{10 N D_{20}}$. [B] Average pupil diameter over time relative to the transition in Experiment 1B (replicating Experiment 1A). The divergence of STEP from its control, CONST, was significant from $600 \mathrm{~ms}$ post-transition. $R E G_{10}-R A N D_{20}$ significantly diverged from its control, $R E G_{10}$, from $860 \mathrm{~ms}$. As in Experiment 1A, no significant differences were observed between $R^{2}{ }_{N D}{ }_{20}-R^{2} G_{10}$ and RAND20 throughout the epoch. The difference between $R E G_{10}-R_{A N D}{ }_{20}$ and $R A N D_{20}-$ $R E G_{10}$ began around $1020 \mathrm{~ms}$ post-transition. $R E G_{10}-R A N D_{20}$ also showed a significantly greater PDR than STEP from $740 \mathrm{~ms}$ onwards. [C, D] Average pupil diameter over time, relative to sequence onset. There were no significant differences between RAND $_{20}$ and $R E G_{10}$ in either experiment. In Experiment $1 B R E_{10}$ showed a significantly larger pupil diameter than CONST between 2880 and 3960ms post-onset. In both experiments, RAND $_{20}$ was associated with a significantly larger pupil diameter than CONST from $2000 \mathrm{~ms}$ (Expt1A) and from 2680ms (Expt1B) post-onset. Importantly there were no differences between $R A N D_{20}$ and $R E G_{10}$ in either experiment. [E] Behavioral hit and false alarm (fa) rates for the gap detection task in Experiment 1A. Grey circles represent individual participant data, and error bars are \pm 1 SEM. Performance on RAND $_{20}$ was significantly reduced relative to the other conditions. [F] Gap detection task in Experiment 1B. Lengthening the gap in Experiment 1B resulted in equated performance across conditions. 


\section{The RAND-REG null effect is not due to pupillary saturation:}

It is important to eliminate the possibility that the lack of PDR to $R A N D_{20}-R E G_{10}$ transitions resulted from pupil diameter increase to saturation during the pre-transition $R A N D_{20}$ part of the sequence. Fig. 2C and D present average pupil diameter in the no-transition stimuli from sequence onset as measured in Exp. 1A and 1B. No significant differences were observed between $\mathrm{RAND}_{20}$ and $R E \mathrm{G}_{10}$ in either Exp. $1 \mathrm{~A}$ or $1 \mathrm{~B}$, suggesting an equivalent average pupil dimeter before the transition. Identical results were also obtained in Exp. 2 and 4 below (see Fig. 4E, 7B), confirming that the absence of a PDR in $R A N D_{20}-R^{2} G_{10}$ transitions cannot be explained based on pre-transition differences between conditions. In Exp. 1 only there appears to be a pre-transition difference between either of the structured (RAND and REG) sequence conditions vs. the CONST condition. It may be due to the fact that the gap detection task was more demanding in the structured relative to the simpler sequences. However, this effect appears to be unstable (not observed with an identical task and stimuli in subsequent experiments see Fig. 4E, 7B) and is therefore not discussed further.

\section{The RAND-REG null-effect is not due to temporal spread of pupil dilation events:}

To confirm that the null effect for $R A N D_{20}-R E G_{10}$ indicates the absence of a pupil response and is not instead a consequence of an increased temporal spread of dilation events, pupil dilation (PD) and constriction (PC) rates were also analyzed (see Methods). This analysis is fundamentally different from the PDR analysis in that it focuses on the incidence of PD (or PC) events, irrespective of their amplitude, and therefore provides a sensitive measure of subtle changes in pupil dynamics potentially evoked by the transitions. Fig. 3 shows pupil dilation events from each trial, for each subject ( $N=32$, combining Exp 1A \& B), over an interval of 2 seconds before to 2 seconds after the transition. 
STEP and $R E G_{10}-R A N D_{20}$ transitions were associated with an increase in PD rate shortly after the transition, whereas no such change in rate was observed for $R A N D_{20}-R E G_{10}$. This effect was also mirrored in the constriction data, confirming that neither PD nor PC dynamics changed following $R A N D_{20}-R G_{10}$ transitions. Results were equivalent across eventduration thresholds of 75 and $300 \mathrm{~ms}$ (see Methods). Overall, this set of analyses provides further evidence for a null PDR response related to the $R A N D_{20}-R E G_{10}$ transition.

To interpret this first set of results, it is important to establish whether the PDR observed for STEP and $R E G_{10}-R_{A N D}$ transitions revealed 'true' sensitivity to pattern violations, or rather was driven by low-level stimulus changes (frequency deviants). In Exp. 1, at least half of $R E G_{10}-R A N D_{20}$ trials involved the appearance of a novel frequency at the time of transition. This is also trivially the case for all STEP trials. It is therefore possible that the PDR reflects a simple response to the detection of a new frequency in the stimulus. In Exp. 2 the stimulus set was amended to include conditions where the transition was manifested as a change in pattern with or without frequency deviants. 
Raster plot of pupil dilation events

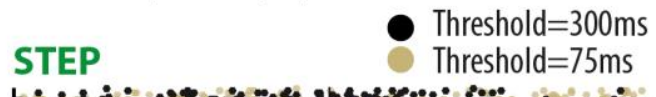

3. CONST

\&f: H. REG $_{10}-$ RAND $_{20}$

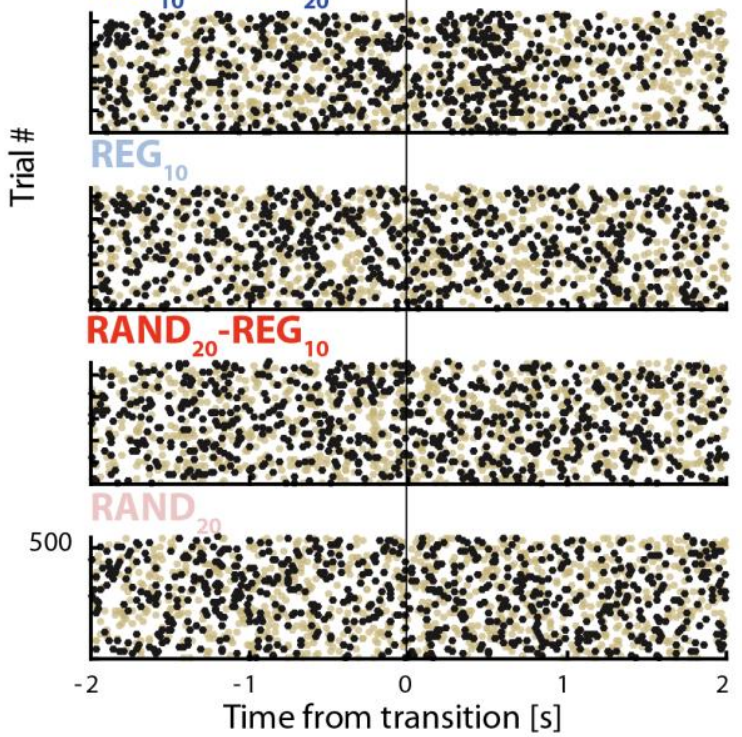

Raster plot of pupil constriction events STEP
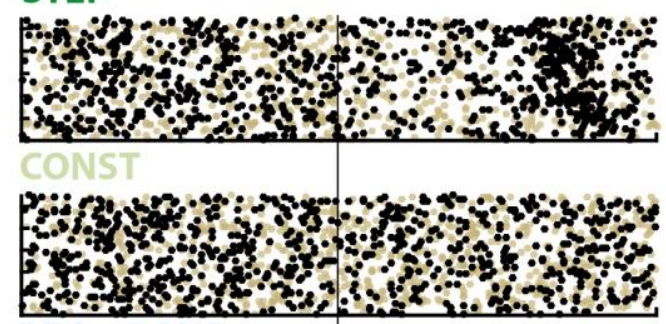

REG $_{10}$-RAND

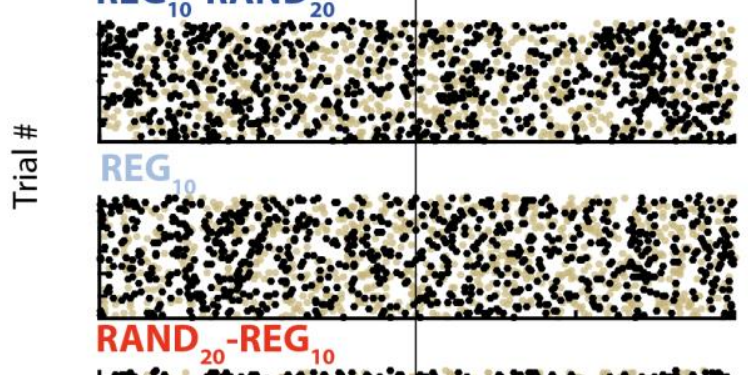

b

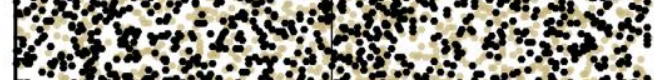

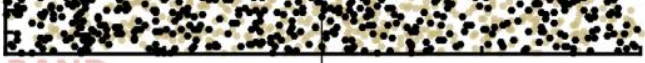

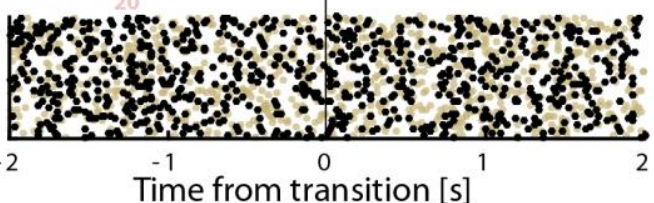

Pupil dilation rate

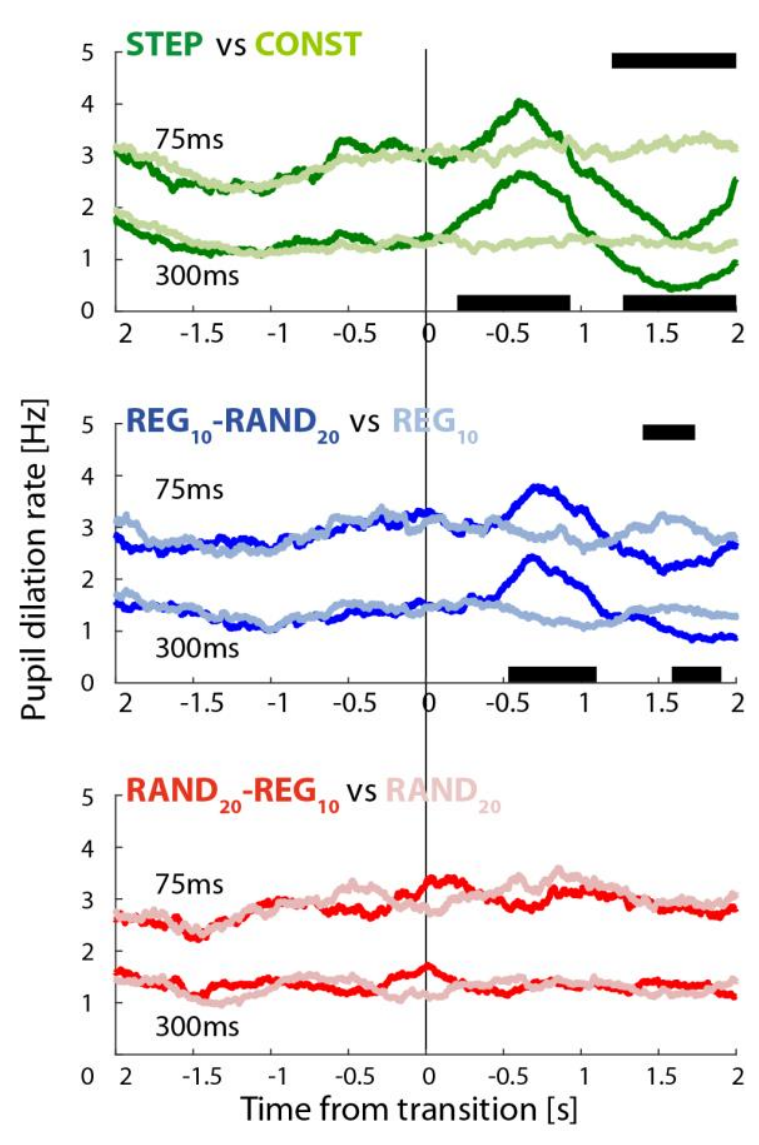

Pupil constriction rate

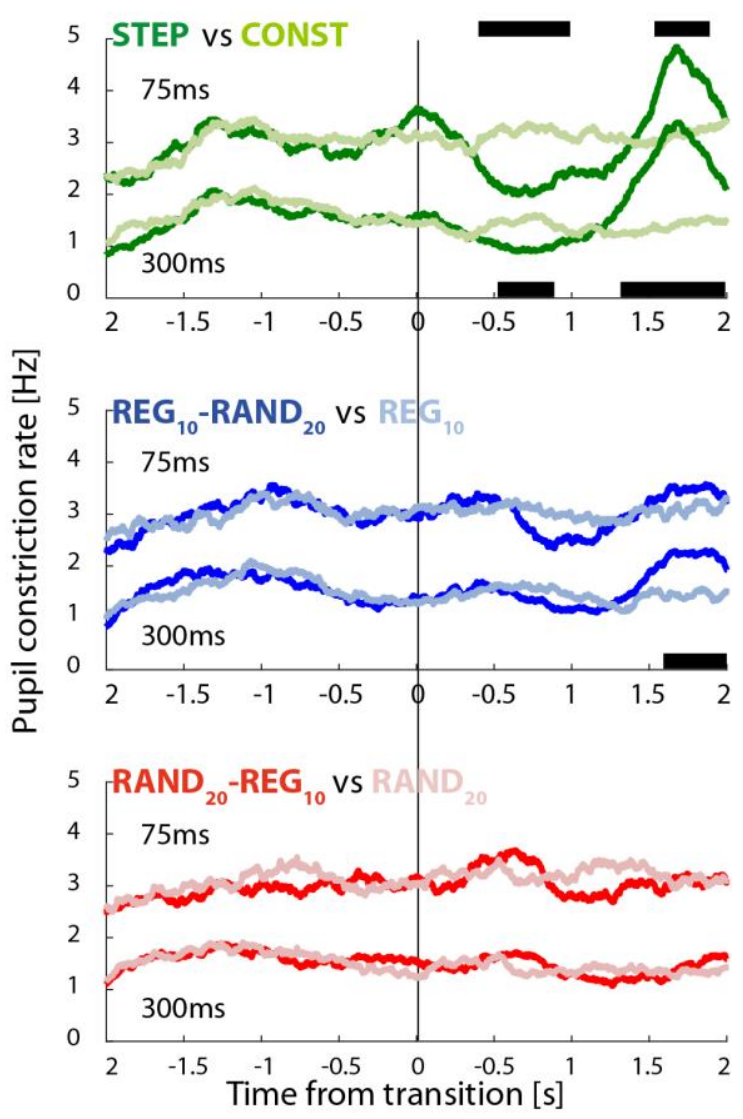


Figure 3: Experiment 1: Pupil dilation and constriction rates. [Top left] Raster plots of pupil dilation (PD) events extracted from all trials and all participants (collapsed over Experiment 1A \& B). Each line represents a single trial. Black dots represent the onset of a pupil dilation with a duration of at least $300 \mathrm{~ms}$, yellow dots represent pupil dilation onsets with a threshold duration of $75 \mathrm{~ms}$. Transition time is indicated by a black vertical line. [Top right] Pupil dilation rate (running average with a 500ms window) as a function of time relative to the transition. The black horizontal lines indicate time intervals where cluster-level statistics showed significant differences between each change condition and its no-change control. The statistics for the PD rates with a threshold duration of $75 \mathrm{~ms}$ and $300 \mathrm{~ms}$ are placed above and below the graph, respectively. [Top] STEP and

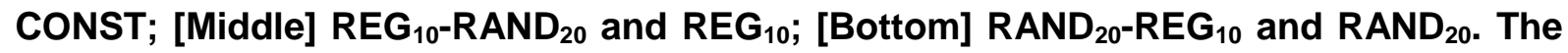
lower panels present the pupil constriction (PC) rate results, arranged in the same format. 


\section{Exp. 2 ( $\mathrm{N}=18)$ : The pupil-linked LC-NE system is activated by 'pure' pattern violations.}

The stimulus set for Exp. 2 is in Fig. 4, top panel. Fig. 4B plots all the conditions which contained a regular-to-random transition: All evoked a marked PDR relative to the $R E G_{10}$ control. Notably a prominent PDR was observed for $R E G_{10}-R A N D_{10}$, i.e. a transition from a REG to a RAND pattern manifested as a change in pattern only, while maintaining the same 10 frequencies. In contrast, no significant difference was observed for any of the random-toregular transitions (Fig. 4D). This was also the case for the $R A N D_{10}-R^{2} G_{10 d}$ condition where the RAND and REG sequences differed in frequency content in addition to the change in pattern. Whilst a small peak is visible in that condition, no significant differences are observed when compared to the no-transition RAND 10 condition (Fig. 4C).

To confirm that the various REG and RAND conditions did not diverge pre-transition, we analyzed the pupil response from stimulus onset (Fig. 4E) and found no significant differences between any of the conditions. Consistent with Exp. 1, behavioral performance did not differ across conditions (Fig. 4F). 


\section{REG}
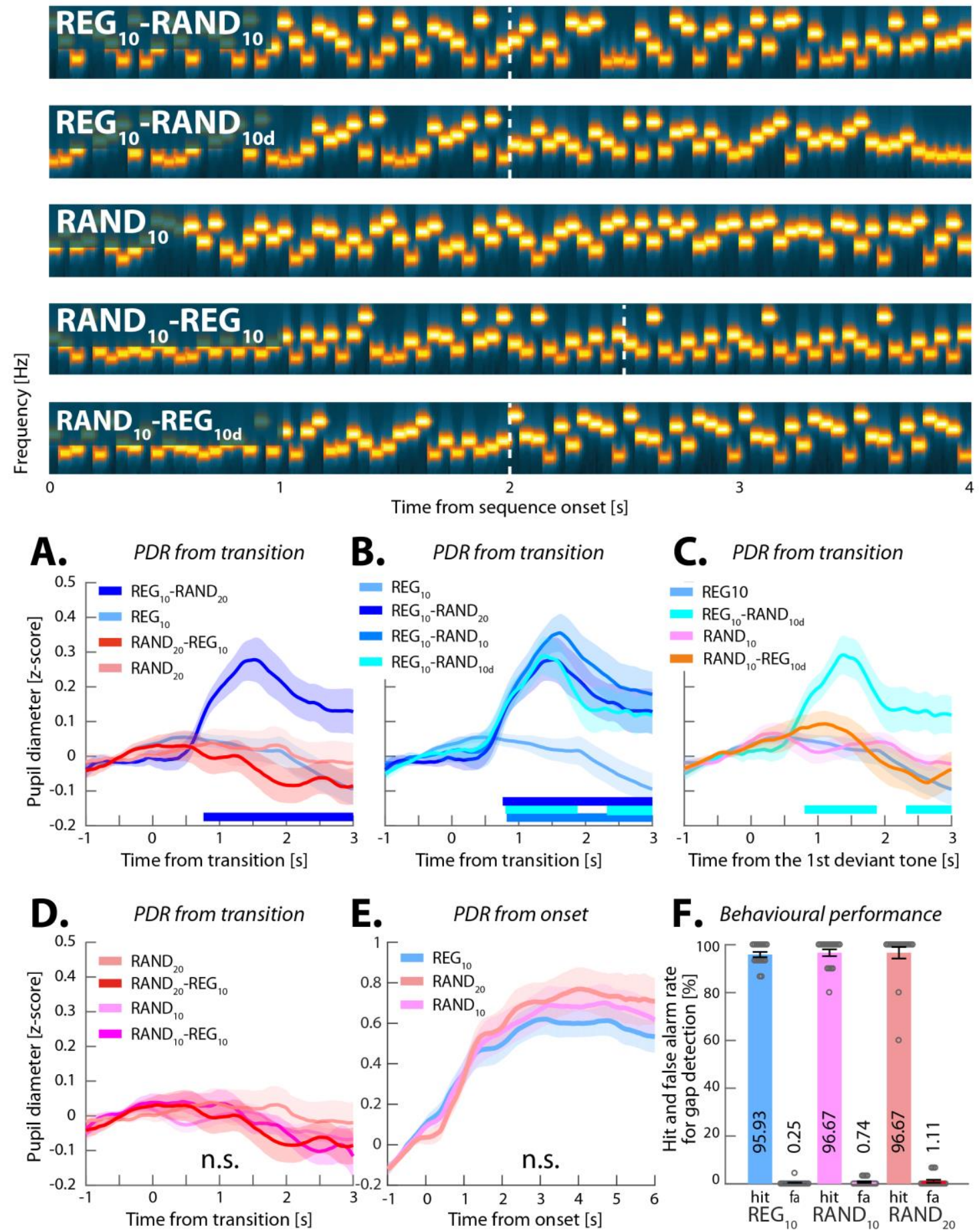

Figure 4: Experiment 2 ( $N=18)$. The PDR response reflects sensitivity to 'pure' pattern violations. [Top] Example spectrograms for the additional stimuli used in Experiment 2. $R E G_{10}$ and $R A N D_{10}$ were generated by randomly selecting 10 frequencies from the pool and then either iterating that sequence to create a regularly repeating pattern or 
presenting them in random order. $R E G_{10}-R_{A N D}{ }_{10}$ and $R A N D_{10}-R G_{10}$ sequences were created from the same 10 frequencies (different sets on each trial). Thus, the transition was manifested as a change in pattern only, without the occurrence of a frequency deviant. $R E G_{10}-R A N D_{10 d}$ and $R A N D_{10}-R G_{10 d}$ were created such that the frequencies used for the REG and RAND portions of the sequence were different (non-overlapping sets of 10 frequencies each). The transition was thus manifested as both a change in pattern, and also as a change in frequency content. The stimulus set also included $R G_{10}-R_{A N D}{ }_{20}, R A N D_{20}-R G_{10}$ and $R A N D_{20}$ sequences (identical to those in Experiment 1). Dashed vertical white lines indicate the transition time. Note that for $R A N D_{10}-R E G_{10}$ the transition time is defined as occurring after the first full regularity cycle (see also Figure 1). The transition time is not adjusted for $R A N D_{10}-R G_{10 d}$ because the transition becomes statistically detectable immediately when the alphabet changes (at the nominal transition time). For presentation purposes, the plotted sequence lengths are equal, but experimental sequences durations varied randomly between 6.0-7.5 s. [Bottom] [A-D] Average pupil diameter over time relative to the transition. Colored horizontal lines indicate time intervals where cluster-level statistics showed significant differences between each change condition and its no-change control. [A] The pupil responses to $R E G_{10}-R A N D_{20}, R A N D_{20}-R E G_{10}$ and their respective controls replicated the pattern observed in Experiment 1: $R E G_{10}-R_{A N D_{20}}$ diverged from $R E G_{10}$ at $780 \mathrm{~ms}$ post-

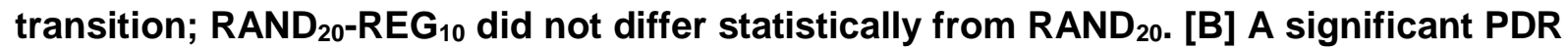
was observed for all conditions containing transitions from $R E G_{10}$. The PDR in $R E G_{10^{-}}$ RAND $_{10}$ increased from $420 \mathrm{~ms}$, and statistically diverged from $R E G_{10} 860 \mathrm{~ms}$ posttransition, peaking at $\sim 1620 \mathrm{~ms}$; the PDR to $R E G_{10}-R^{2} N_{10 d}$ diverged from $R E G_{10}$ at $800 \mathrm{~ms}$, peaking at $1380 \mathrm{~ms}$. [C] A comparison between the two conditions which

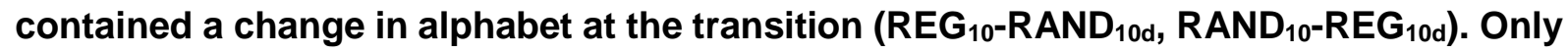
REG $_{10}-$ RAND $_{10 \mathrm{~d}}$ evoked a PDR [D] None of the stimuli containing transitions from RAND $_{10}$ evoked a significant PDR. [E] Average pupil diameter over time, from stimulus onset. No differences were observed between any of the conditions. [F] Behavioral results for the gap detection task. Error bars are \pm 1 SEM; grey circles represent individual participant data. Performance was at ceiling. 


\section{Experiment $3(\mathrm{~N}=14)$ : The effect of active transition detection}

Exp. 1 and 2 measured responses to transitions when they were not behaviorally relevant. To understand the effect of task relevance on PDRs, we introduced an active behavioral transition-tracking task. The experimental conditions were as in Exp. 1, but participants were asked to detect pattern changes rather than silent gaps.

Behavioral results are summarized in Fig. 5A. Hit rate data demonstrated that all transition conditions were highly detectable by human listeners. Although false positive rates were all low, there was a main effect of condition $(F(1.405,18.262)=15.272, p<0.001)$, where, consistent with previous work (Barascud et al., 2016), there was a small but significantly higher false positive rate for (no-transition) $\mathrm{RAND}_{20}$ compared with CONST and $\mathrm{REG}_{10}$, with the latter two not differing significantly ( $p=0.083$; Bonferroni corrected). To avoid confounds due to false positive disparities, all false positive trials were excluded from pupil analysis.

For reaction time, a repeated measures ANOVA confirmed a main effect of condition $(F(2,26)=90.723, p<0.001 ;$ STEP $<$ REG-RAND $<$ RAND-REG), consistent with previous work (Barascud et al., 2016).

Turning to Pupillometry, we observed three fundamental differences relative to Exp 1:

\section{(1) RAND-REG also evoked a PDR response}

Fig. 5B plots the average normalized pupil diameter relative to the transition. Clear PDRs were observed in all three change conditions. Critically - and unlike the previous experiments with a gap detection task - a robust PDR was associated with $R A N D_{20}-R G_{10}$ when the transition was task-relevant.

\section{(2) Delayed PDR peak for $R E G_{10}-R A N D_{20}$}

Compared to Exp. 1 and 2, we also observed a substantial shift in the latency of the PDR to REG ${ }_{10}-$ RAND $_{20}$ (Fig. 5C). Active transition detection slowed the PDR by $\sim 300 \mathrm{~ms}$, with 
a peak latency of $1840 \mathrm{~ms}$ in Exp. 3 relative to $1400-1600 \mathrm{~ms}$ in the previous experiments. Peak latency to STEP did not change.

\section{(3) Differences in pupil diameter observed from sequence onset}

In Exp. 1 and 2 (see also Exp. 4, below) we consistently observed no difference between $R E G_{10}$ and $R A N D_{20}$ when analyzing pupil responses relative to sound onset. In contrast, here in Exp 3 we observed a pre-transition disparity between $R E G_{10}, R_{A N D_{20}}$ and CONST (Fig. 5D), such that $\mathrm{RAND}_{20}$ sequences evoked the largest sustained pupil dilation, followed by $R E G_{10}$. The sustained pupil diameter for $R A N D_{20}$ was significantly higher than $R E G_{10}$ from 3080 ms post-onset. This cannot be explained by the higher false alarm rate of RAND $_{20}$, as trials with incorrect responses were excluded from analysis, but may be a consequence of the computational demands or perceptual effort associated with tracking RAND $_{20}$ sequences (see discussion). 
A.

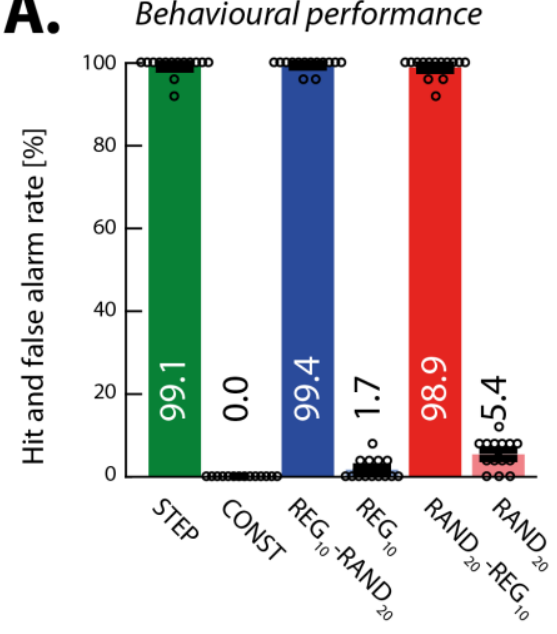

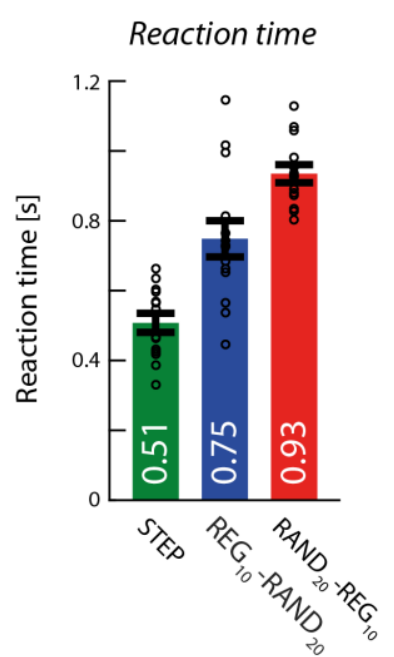
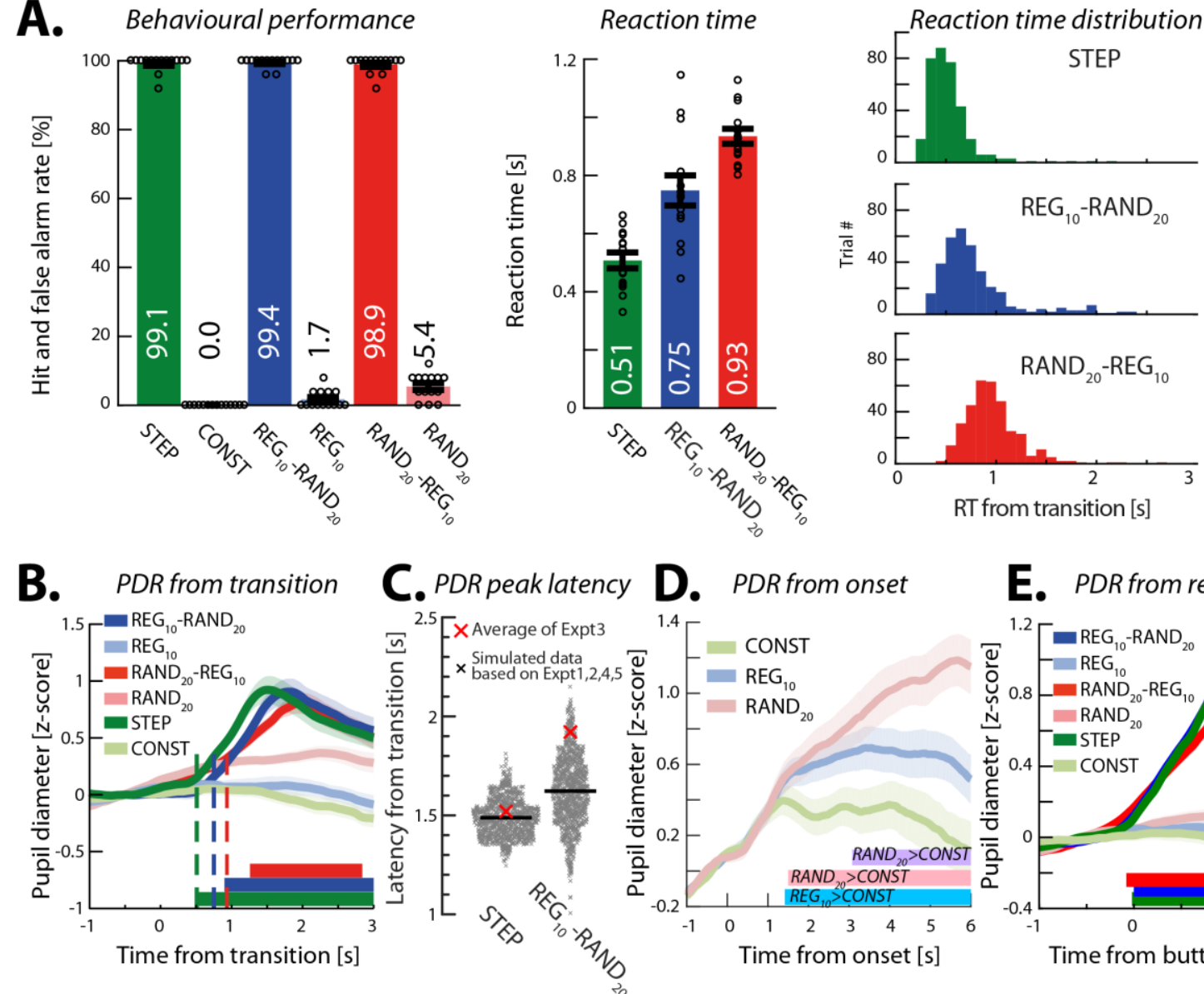

E. PDR from response

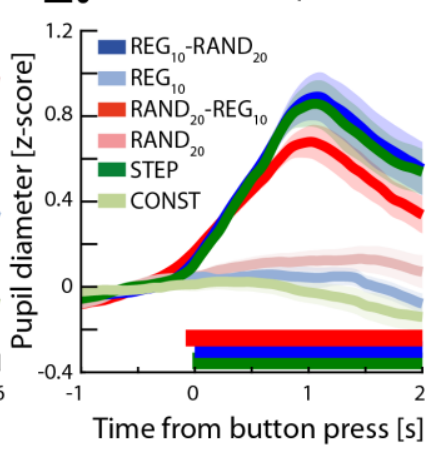

Figure 5: Experiment 3 ( $\mathrm{N}=14)$ : Active transition detection. [A] Behavioral performance (active transition detection). [Left] Hit rates and false positive rates. Circles indicate individual subject data; error bars are \pm 1 SEM. [Middle] Reaction times (RT). [Right] The distribution of RTs (across trials and participants) for each transition condition. The variance of the RT distribution of $R A N D_{20}-R E G_{10}$ was smaller than that of $R E G_{10}-R{ }^{2} D_{20}$ (Levene's test, $F(1,690)=14.426, p=0.0002)$. [B] Average pupil diameter relative to the transition. Solid lines represent the average normalized pupil diameter, relative to the transition. Shading shows \pm 1 SEM. Colored horizontal lines indicate time intervals where cluster-level statistics showed significant differences between each change condition and its control. Dashed vertical lines mark the average RT for each condition. Clear PDRs were observed for all 3 transitions. The PDR to STEP increased from $\sim 490 \mathrm{~ms}$ post-transition, peaking at $1500 \mathrm{~ms}$; it statistically diverged from CONST from $540 \mathrm{~ms}$ through to sequence offset. For $R E G_{10}-R A N D_{20}$, the responses commenced $\sim 750 \mathrm{~ms}$ post-transition, peaking at $1840 \mathrm{~ms}$, and statistically diverged from REG at $900 \mathrm{~ms}$ post-transition through to sequence offset. For $R A N D_{20}-R G_{10}$, the response started at $930 \mathrm{~ms}$, peaked at $1980 \mathrm{~ms}$, and statistically diverged from its control RAND 20 between 1260 and $2840 \mathrm{~ms}$. Comparing transition conditions directly, no difference was

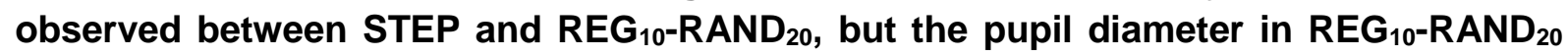
became significantly greater than that in $R A N D_{20}-R G_{10}$ between 700 and $1480 \mathrm{~ms}$ posttransition. [C] A comparison of PDR peak latency to STEP and REG ${ }_{10}-R^{2}{ }^{2} D_{20}$ in Experiment 3 relative to Experiments 1,2,4,5 (see methods). The scatterplots show the distribution of the simulated peak latency of STEP (left) and REG ${ }_{10}-R_{A N D_{20}}$ (right) in the 
'gap detection' experiments. The red crosses indicate the mean peak latency in Experiment 3. The results showed no difference $(p=0.338)$ for STEP, but a greater latency for $R E G_{10}-R_{A N D}(p=0.048)$. [D] Average pupil diameter over time relative to the sequence onset. RAND 20 and $R E G_{10}$ statistically diverged from CONST at 1500 and $1420 \mathrm{~ms}$ post-onset, respectively. Interestingly, RAND ${ }_{20}$ evoked an even larger PDR than $\mathrm{REG}_{10}$ from $3080 \mathrm{~ms}$ post-onset. [E] Average pupil diameter over time, relative to button press. 
We explored the relationship between reaction time (RT) and pupil diameter across single trials (Fig. 6). Generally, peak pupil dilation (indicated by hot colors in Fig. 6A) occurred about 1 second after the button press (Einhäuser et al., 2010). This relationship is also evident in Fig. 5E: the onset of the PDR to each of the transitions closely coincides with button press timing.

The fact that peak PDR occurs substantially after the button press may indicate that the behavioral response itself either triggers or otherwise modulates the PDR. To understand the relationship between RT and the PDR, we examined whether button press timing was systematically linked to key measures of PDR dynamics. We tested the relationship between RT and the maximum PDR amplitude (Fig. 6B) on a single-trial basis. (All subsequent analyses are rank-based, with subjects as random effect and reaction time as fixed effect (Bates et al., 2014, see Methods)). In the STEP condition, there was no significant association between RT and maximum PDR amplitude $(\mathrm{t}(84.86)=1.29, \mathrm{p}=0.1996)$; this also held true in the $R E G_{10}-R A N D_{20}$ condition $\left(t(234.6)=0.22, p=0.8232\right.$ ). However, in the $R A N D_{20}-R G_{10}$ condition, RT was significantly associated with maximum PDR amplitude $(\mathrm{t}(334.9)=3.06, \mathrm{p}=$ 0.0024): here, RT was estimated to account for $2.8 \%$ of variance in the maximum PDR amplitude (estimate of partial $\mathrm{R}^{2}$ derived using an implementation of the Nakagawa and Schielzeth (2013) algorithm, see Methods).

We then asked whether RT was associated with the timing of the maximum PDR (Fig. 6C). In the STEP condition, there was a small but significant association between RT and PDR latency $(\mathrm{t}(297)=1.97, \mathrm{p}=0.0496$, accounting for an estimated $1.5 \%$ of PDR latency variance. (Note, however, this association was not significant when slopes were allowed to vary). In the $R E G_{10}-R A N D_{20}$ condition, $R T$ was also significantly associated with PDR latency $(t(308.8)=4.64, p<0.0001)$, and accounted for an estimated $7.2 \%$ of its variance. Finally, RT was also associated with PDR latency in the RAND $20-\mathrm{REG}_{10}$ condition $(\mathrm{t}(310.8)=4.85, \mathrm{p}<$ 0.0001 ), and an estimated $6.3 \%$ of RT variance. 
Finally, we asked if RT was associated with the timing of the maximum derivative of the PDR (i.e. the time at which the rate of change of pupil size is maximal; Fig. 6E). As with the other two dependent variables, reaction times in STEP were not associated with the maximum derivative PDR latency, $\mathrm{t}(129.5)=0.50, \mathrm{p}=0.6180)$. Nor was there a significant association in the REG ${ }_{10}-R_{A N D}$ condition $\left.(t(115.6)=-0.20, p=0.8415)\right)$. However, $R T$ in the $\mathrm{RAND}_{20}-\mathrm{REG}_{10}$ condition was associated with the maximum derivative PDR latency, $t(328.9)=2.44, p=0.0151)$, and $1.8 \%$ of its variance.

In sum, the pattern of associations between participants' reaction times and various pupil measures suggests that the amplitude and timing of the pupillary response to both $\mathrm{RAND}_{20}-\mathrm{REG}_{10}$ and $\mathrm{REG} \mathrm{G}_{10}-\mathrm{RAND}_{20}$ are related to button press timing, but only to a modest degree, with RT accounting for between $\sim 2-6 \%$ of estimated variance. While these analyses are limited by the relatively small amount of trials per condition/subject, this outcome suggests that the appearance of a PDR to the RAND $20-R E G_{10}$ transition in Exp 3 is not primarily driven by the button press. Rather, having listeners actively monitor and respond to the statistical transitions prompted a change in the underlying cognitive process, e.g. by delineating the category (or decision) boundary between RAND and REG, and thereby rendering the transition as a model violation (see Discussion). 


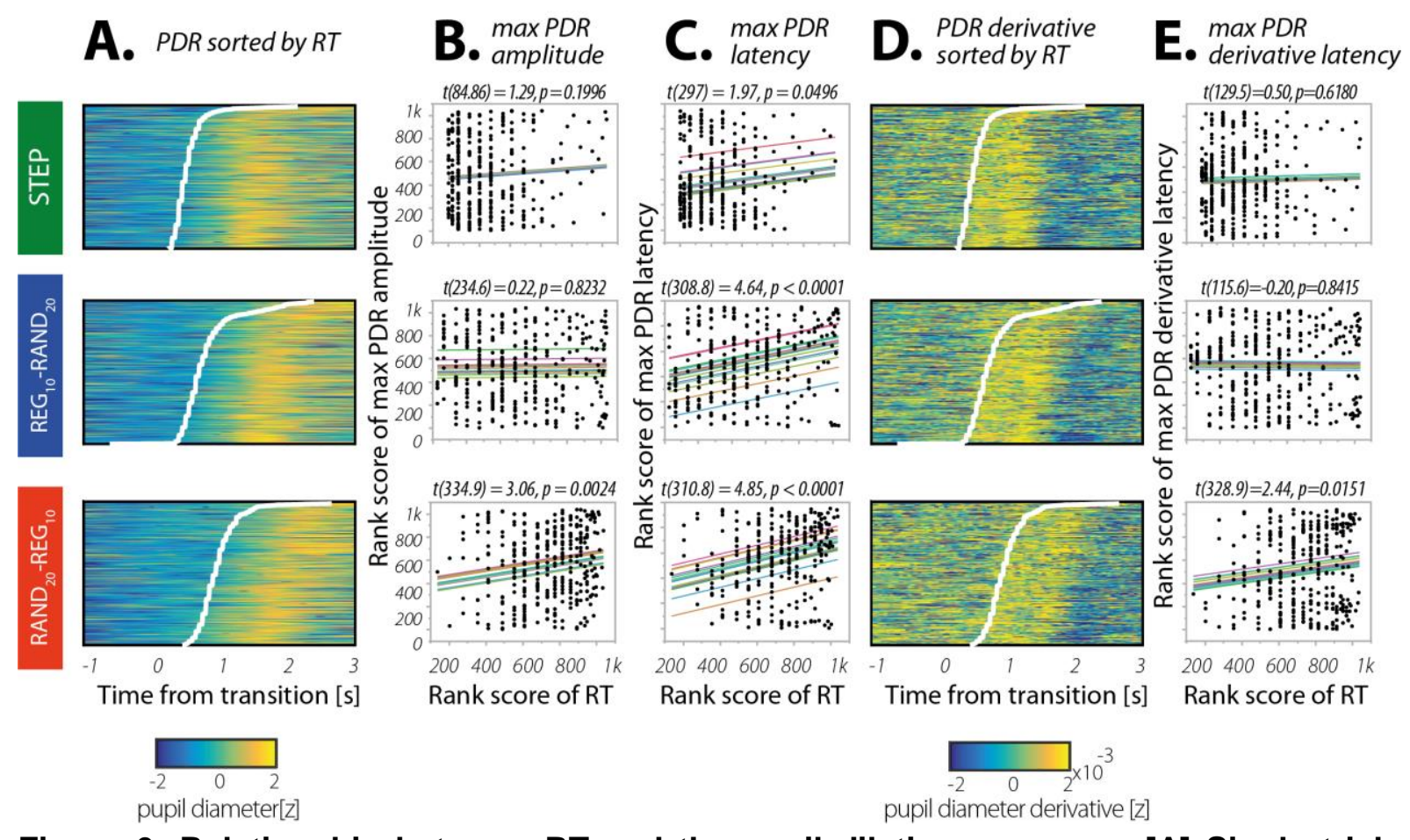

Figure 6: Relationship between RT and the pupil dilation response. [A] Single trials sorted by $R T$ ( $y$-axis, $R T$ indicated by white lines) shown against the time relative to the transition (x-axis) with the colors showing pupil diameter (the warmer the color, the larger the pupil). [B] Scatter plots show the maximum PDR amplitude for each trial (ranked low to high on the $y$-axis) versus ranked $\mathrm{RT}$ for the same trial (x-axis), separated by condition as in [A]. Each line of fit shows the modelled random effect of subject (offset), with slope the fixed effect of RT. Fixed-effects t-values and associated p-values appear above each fitted scatterplot. [C] Rank maximum PDR latency vs. RT. Scatterplot and fitting as in [B]. [D] Single trials sorted by RT (y-axis, RT indicated by white lines) shown against the time relative to the transition ( $x$-axis) with the colors showing the rate of change of pupil diameter (the warmer the color, the larger the rate of change in pupil size). [E] Rank maximum derivative latency vs rank RT (scatterplot fits as in [C]). 


\section{Exp 4: Pupil responses to transitions from randomness}

We have argued that to achieve effective model maintenance, the brain must arbitrate between gradual and punctate environmental changes. In other words, at each point in time, the brain must decide whether to continue updating its current representation of environmental contingencies or instead abandon the existing model and prioritize bottom-up evidence accumulation ("out with the old, in with the new"). Our results thus far suggest that what determines the difference between gradual and abrupt change can be gleaned through delineating the contingencies which evoke a PDR.

In REG-RAND, and trivially so in STEP, the statistical violation is immediately observable if listeners form a robust representation of the patterning of the REG sequences This could therefore be sufficient to trigger the abrupt-model violation signal.

By contrast, since absolutely any sequence of tones has the same probability under RAND, the detection of transitions out of this distribution is statistically more complicated. The lack of a PDR for $\mathrm{RAND}_{20}-\mathrm{REG}_{10}$ transitions, when not behaviourally relevant, is taken to indicate that that this transition is indeed not treated as an abrupt model violation. In Exp 4 we explore whether the same is true for less complex regular patterns. 

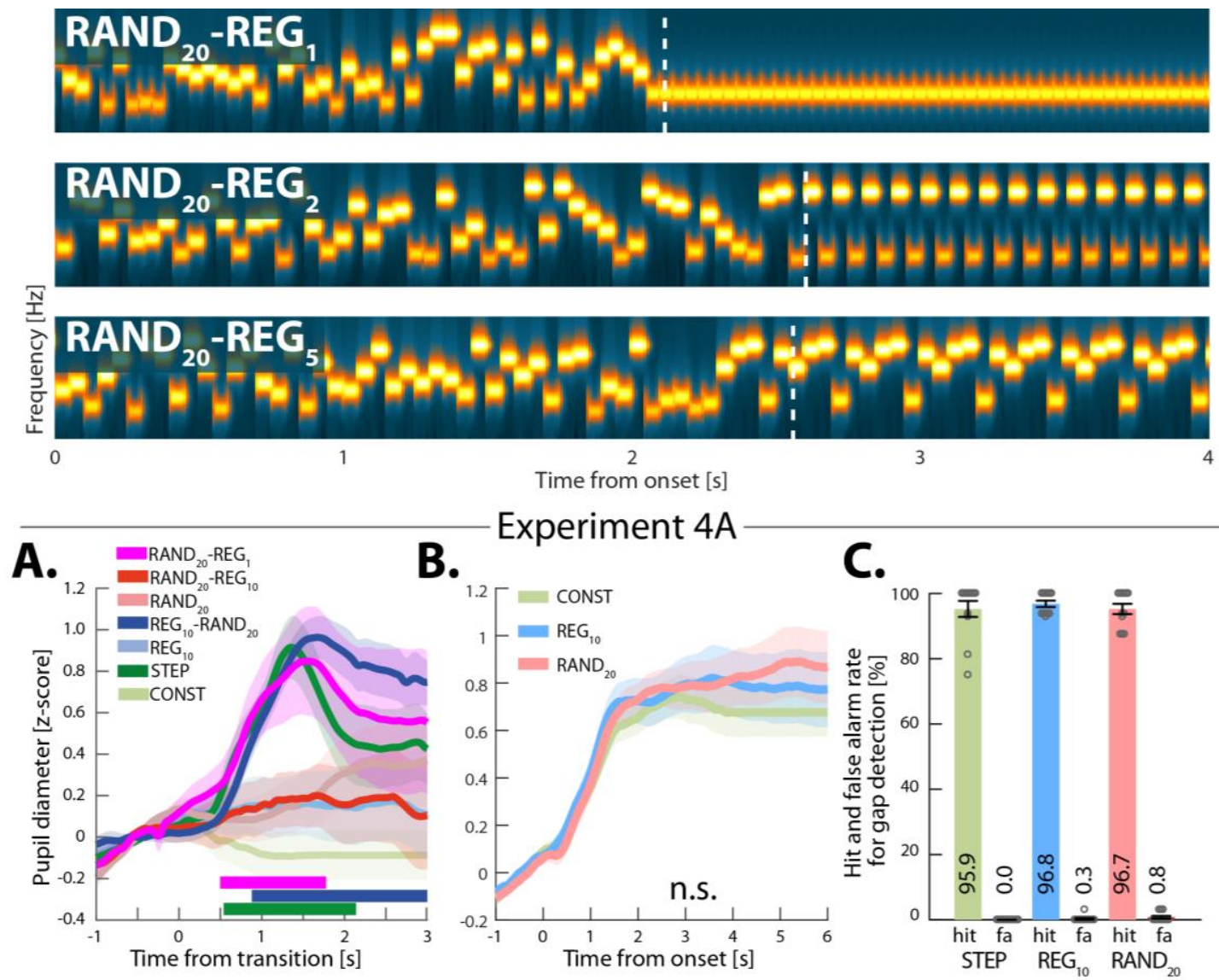

B.

Experiment $4 \mathrm{~A}$
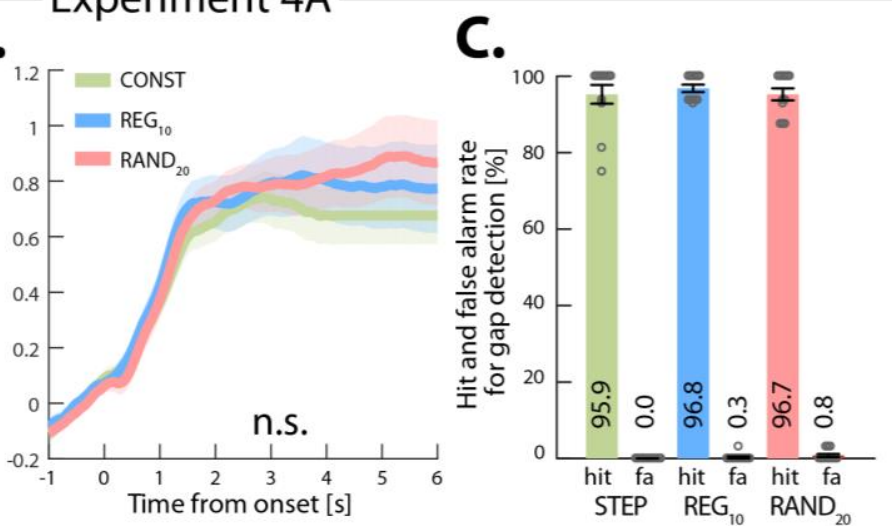

Experiment 4B
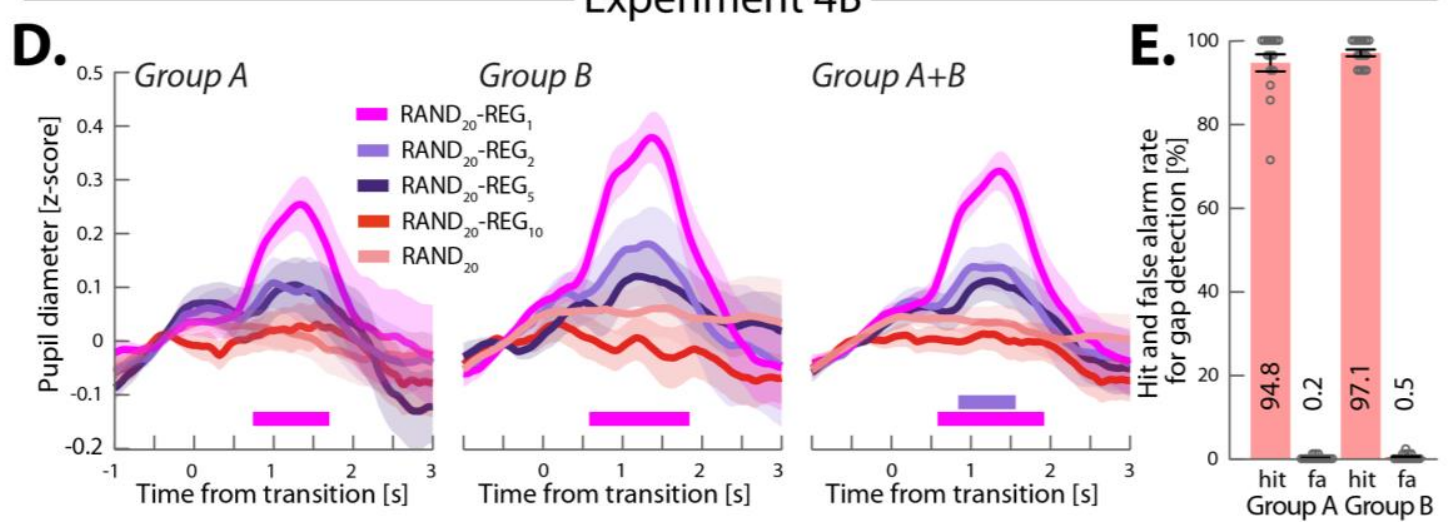

Figure 7: Experiments 4A ( $\mathrm{N}=12)$ and $4 \mathrm{~B}$ (two groups of $\mathrm{N}=15)$. Abrupt reduction in the PDR for regularities more complex than $R G_{1}$. [Top] Example spectrograms for the additional stimuli used in Experiments $4 A$ and $B$. RAND ${ }_{20}-R E_{1}$ consisted of a transition from a random sequence (generated by sampling frequencies from the full pool with replacement $R A N D_{20}$ ) to a single repeating tone. $R A N D_{20}-R E G_{2}$ consisted of a transition from a RAND R $_{20}$ sequence to a regular pattern consisting of two randomly selected tones. $\mathrm{RAND}_{20}-\mathrm{REG}_{5}$, consisted of a transition from a RAND 20 sequence to a regular repeating pattern consisting of 5 tones. Dashed vertical white lines indicate the transition time, defined as occurring after the first full regularity cycle. For presentation purposes the plotted sequence lengths are equal. Actual durations varied randomly between 6 to 7.5s.

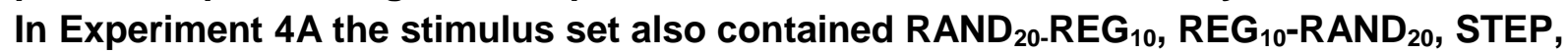
REG $_{10}, R_{A N D_{20}}$ and CONST sequences. In Experiment 4B the stimulus set additionally contained RAND $20-R_{20}{ }_{10}$ and RAND $_{20}$ sequences. [Bottom] [A] Average pupil diameter 
relative to the transition in Experiment 4A. Solid lines represent the average normalized pupil diameter, relative to the transition. The shaded area shows $\pm 1 \mathrm{SEM}$. Colored horizontal lines indicate time intervals where cluster-level statistics showed significant differences between each change condition and its control. A robust PDR was evoked by the transition in $\mathrm{RAND}_{20}-\mathrm{REG}_{1}$, becoming significant from $500 \mathrm{~ms}$ post-transition, at approximately the same time as STEP, and lasting through to $1780 \mathrm{~ms}$. The data also replicate the general pattern in Experiments 1 and 2: a PDR evoked by $R \mathrm{G}_{10}-\mathrm{RAND}_{20}$

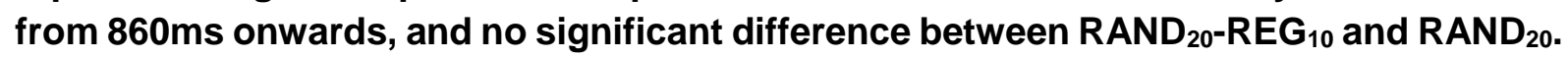
The transition conditions were also compared directly and revealed a significant difference between $R E G_{10}-R{ } N_{20}$ and $R A N D_{20}-R E G_{10}$ from $\sim 1020$ ms post-transition. [B] Average pupil diameter over time from stimulus onset. No differences were observed between any of the conditions. [C] Behavioral results for the gap detection task in Experiment $4 \mathrm{~A}$ with \pm 1 SEM error bars, and grey circles representing individual participant data. There was no statistical difference between conditions. [D] Average pupil diameter over time relative to the transition in Experiment 4B: [Left] Group $A$ $(\mathrm{N}=15)$. A clear PDR is observed for $R A N D_{20}-R E G_{1}$ which diverged from its control, RAND $_{20}$, from 740 to $1700 \mathrm{~ms}$. No significant differences were observed in the other conditions. [Middle] Group $B(\mathrm{~N}=15)$. $R A \mathrm{RD}_{20}-\mathrm{REG}_{1}$ diverged from $\mathrm{RAND}_{20}$ between

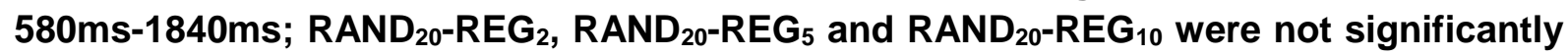
different from RAND ${ }_{20}$. [Right] both groups combined $(\mathrm{N}=30)$. Significant PDRs were observed for RAND ${ }_{20}-\mathrm{REG}_{1}$ (from 580 to $1920 \mathrm{~ms}$ ) and RAND ${ }_{20}-\mathrm{REG}_{2}$ (from 840 to $1560 \mathrm{~ms}$ ) transitions only. [E] The behavioral performance for both groups was at ceiling. 


\section{$\operatorname{Exp} 4 A(N=12)$}

We asked whether the most basic regular pattern - a single repeating tone $\left(R E G_{1}\right)$ evokes a PDR. Naive listeners (performaing a gap detection task) were presented with RAND $_{20}-\mathrm{REG}_{1}$ sequences (Fig. 7, top panel), in addition to STEP, REG $10-\mathrm{RAND}_{20}$ and $\mathrm{RAND}_{20}-\mathrm{REG}_{10}$. Replicating the result of Exp 1, we observed a PDR to $R E G_{10}-R_{A N D} 20$ but not to $R A N D_{20}-R E G_{10}$. By contrast, the $R A N D_{20}-R E G_{1}$ transition evoked a fast-onset and robust PDR of similar amplitude to that evoked by transitions from regularity to randomness. This result is consistent with previous demonstrations that the violation of randomness by repetition evokes an MMN-like response (Horváth and Winkler, 2004; Rosburg, 2004) - a finding which was taken to suggest that the auditory system represents stochastic frequency variation as a regularity per se (Wolff and Schröger, 2001).

As with the previous experiments, there was no significant difference between $R_{E G} G_{10}$ and $\mathrm{RAND}_{20}$ pre-transition (Fig. 7B), and no behavioral difference in the gap detection task across the three conditions (Fig. 7C).

\section{Exp 4B (two groups of $N=15$ )}

How does the PDR evolve as the regularity becomes more complex, e.g., as we add more elements to the regular pattern? In Exp $4 B$, in addition to $R A N D_{20}-R E G_{1}$, we also included transitions from $\mathrm{RAND}_{20}$ to repeating 2-, 5-, and 10-tone patterns $\left(\mathrm{REG}_{2}, \mathrm{REG}_{5}, \mathrm{REG} \mathrm{G}_{10}\right.$, Fig. 7 top panel, $\mathrm{REG}_{10}$ not shown).

Due to the increased number of conditions and therefore longer experiment time, data were noisier than in the previous experiments. Thus, to replicate the effects from the first group (group $A ; N=15$ ), the experiment was repeated in another group of listeners (group $B ; N=15$ ). We also collapsed the data across both groups to maximize statistical power.

For both groups, the behavioral performance in the gap detection task was at ceiling (Fig. 7E). Fig. 7D plots the results of Exp 4B for each group separately, and when pooled 
together. In each group, and as in Exp 4A, RAND $20-R E G_{1}$ evoked a significant PDR; however, a clear PDR was not observed for the other conditions. The average normalized pupil diameter in $R A N D_{20}-R E G_{2}$ and $R A N D_{20}-R E G_{5}$ appeared to suggest a small (non-significant) gradual increase between $640 \mathrm{~ms}$ and $800 \mathrm{~ms}$, which might indicate the presence of a sub-threshold effect. Indeed, collapsing the data across groups did demonstrate a significant PDR for $\mathrm{RAND}_{20}-\mathrm{REG}$, between $840-1560$ ms post-transition. The PDR for $\mathrm{RAND}_{20}-\mathrm{REG}_{5}$ remained non-significant. These effects are also mirrored in the PD rate analysis (see sup. Materials).

Overall the results of Exp 4B demonstrate a sharply reduced PDR for regularities more complex than $R E G_{1}$ : While $R E G_{1}$ consistently evoked a robust response in both groups $A$ and $B$, and with an amplitude identical to that observed for STEP and $R E G_{10}-R A N D_{20}$ (Exp 4A, Fig. $7 A$ ), the PDR to $R A N D_{20}-R E G_{2}$ was substantially reduced, such that it required double the power to reach significance. The small effect of $R E G_{2}$ may indicate that for some subjects or in a subset of trials a PDR was present. The lack of a PDR for more complex regularities suggests that the associated transitions are treated as a gradual rather than abrupt transition with respect to the internal model maintained for $\mathrm{RAND}_{20}$.

\section{General Discussion}

We report two main findings: First, when pattern changes were behaviorally irrelevant, pupil dilation responses (PDR) were evoked exclusively by changes associated with violations of regularity. Second, behavioral relevance exerted a major effect on pupil dynamics, changing the responses both during the establishment of patterns, and at transition periods.

Research in animal models has established a robust link between phasic pupil responses and spiking activity within the LC, providing compelling evidence for pupil dynamics as an indirect measure of NE release (Joshi et al., 2016). Our observations are therefore 
interpreted in the context of understanding the role of the pupil-linked LC-NE system in reporting on aspects of the statistics of rapid sensory signals. The results are consistent with a hypothesized role of NE as a model interrupt signal, and provide a rich view of the contingencies that have automatic and/or controlled access to this interrupt.

A large body of work has suggested a gating role for NE in balancing bottom-up-driven sensory processing vs. top-down priors (Bouret and Sara, 2005; Dayan and Yu, 2006; Sara and Bouret, 2012; Yu and Dayan, 2005). Direct electrophysiological recording in animal models (Aston-Jones et al., 1997; Bouret and Sara, 2004; Sara and Segal, 1991; Vankov et al., 1995) and fMRI in humans (Payzan-LeNestour et al., 2013) have observed neural activity in LC in response to unexpected and abrupt contextual changes. Pharmaceutical evidence has demonstrated that downregulating NE results in impaired adaptation to environmental changes (Marshall et al., 2016; McGaughy et al., 2008) whereas pharmacologically stimulating the noradrenergic system is associated with increased learning rates (Devauges and Sara, 1990; Howlett et al., 2017). Indirect measures of NE release, based on pupillometry, have also revealed an association between NE signaling and increased learning rates - a proxy for model resetting (Krishnamurthy et al., 2017; Nassar et al., 2012). However, the existing literature is limited by the fact that most of the experimental results taken to support the 'NE as an interrupt signal' hypothesis have involved tasks in which inputs evolve slowly over time and participants make either active decisions about stimulus predictability or are required to form stimulusresponse associations (Jepma and Nieuwenhuis, 2011; Krishnamurthy et al., 2017; Lavín et al., 2014; Marshall et al., 2016; Nassar et al., 2012; Payzan-LeNestour et al., 2013; Preuschoff et al., 2011). Here we demonstrate that NE also plays a role in coding model violations on time scales relevant to tracking unfolding sensory information, even when it is not behaviorally relevant. 


\section{PDR specificity under behaviorally-irrelevant listening conditions.}

Deviants or salient changes in sound sequences are well known to evoke PDRs, even under passive listening conditions (Liao et al., 2016; Nieuwenhuis et al., 2011). These observations have prompted a suggestion that, as part of a broader 'fight or flight' response, pupil activation reflects the operation of an interrupt signal that halts current ongoing activities to allow an attentional shift towards the new event, thus facilitating adaptive behavior (Nieuwenhuis et al., 2011; Wang and Munoz, 2015). Phasic LC-NE activation has duly been hypothesized to serve as a neural interrupt signal for unexpected events (Bouret and Sara, 2005; Dayan and Yu, 2006; Payzan-LeNestour et al., 2013), prompting the resetting of topdown connectivity when sensory information indicates that the currently instantiated model of the environment is no longer valid. These observations raise the obvious - and behaviorally important - question of what exact changes are able to drive the interrupt signal.

One common idea is that sensory processing involves rich statistical modeling (Friston, 2005; Knill and Richards, 1996; Rao, 2005), which suggests that many changes might drive the interrupt signal. However, not only are there obvious dangers to making interruption too promiscuous when neural processing is focusing on a task for which the statistics are irrelevant, but it is also computationally costly to build detailed models of complex signals when these do not matter. This consideration motivated a heuristic separation between expected and unexpected uncertainty (Dayan and Yu, 2006; Yu and Dayan, 2005) with events falling in the latter category triggering model interruption, allowing a more sophisticated model-building process to occur, if appropriate.

Here, we exploited the statistical asymmetry between REG-RAND and RAND-REG to gain some further insight into these limits. The transition in REG-RAND is simple to detect, given knowledge of REG. However, for the RAND-REG transition, the RAND model is not directly falsified because all tones within the REG pattern are strictly consistent with the RAND model. The hypothesis is therefore that they are treated in terms of expected - rather than 
unexpected - uncertainty, leading to gradual rather than abrupt model change. In line with the formulation proposed by Dayan \& Yu (2006), we found that, when the transition was irrelevant to the behavioral goal, the LC-NE system appeared to ignore RAND-REG transitions. This was observed for even moderately complex REG patterns, and even though listeners - and their brain activity - readily detect them on a similar time scale to REG-RAND transitions. This not only refutes the suggestion that any perceptually salient and contextually novel set of observations can drive NE, but also hints at limits to the statistical model-building process.

It is important to note that our stimuli were matched spectrally, and in terms of behavioral detectability and time to process, and were presented at a rate beyond that at which listeners can actively track structural content (Warren, 2008). Thus we extend to the preattentive case findings about NE and interrupts originally derived from decision-making tasks and demonstrate that the pupil-linked LC-NE system plays an obligatory role in tracking the statistics of unfolding sensory input.

The sharp drop in the PDR in $R A N D_{20}-R E G_{2}$ relative to $R A N D_{20}-R E G_{1}$ (Fig. 7D), indicates that, while the transition in $R A N D_{20}-R E G_{1}$ is coded explicitly as an abrupt model violation, the transition in $\mathrm{RAND}_{20}-\mathrm{REG}_{2}$ - from a random to a two-tone pattern - is not. One suggestion is that the brain engages in a form of automatic latent model building using just the last few tones. If the latent model based on those few tones fits them much better than the prevailing model, then an abrupt change is reported. Under this hypothesis, the fact that even as simple a sequence as two alternating tones does not generally lead to model change detection suggests stringent constraints on the automatic model construction - perhaps that it encompasses no more than two successive tones. It is tempting to speculate that such model construction could be implemented by low-level coding mechanisms e.g. adaptation or repetition suppression, both of which would lead to detectably unusual patterns of activity in tonotopically organized neural populations. 
Importantly, we interpret the abrupt difference between $R A N D-R E G_{1}$ and $R A N D-R E G_{2}$ (despite their perceptual and statistical similarity) as further evidence for the specificity of the PDR for punctate changes in stimulus statistics. Critically, this specificity can be reversed when transitions are behaviorally relevant (Exp. 3; see more below).

\section{Relationship to brain responses}

The observed PDR specificity is consistent with patterns of brain responses measured with Electro- and Magneto-encephalography (EEG, MEG) in naïve, distracted listeners (Fig. 1B). Robust brain responses are observed to both RAND-REG and REG-RAND transitions, but importantly, the response dynamics are distinct, revealing the differing computational demands of each transition type: RAND-REG transitions evoke a progressive increase in sustained brain responses, hypothesized to reflect the gradual increase in model precision associated with the increased predictability of the REG patterns (Barascud et al., 2016). This rise in the sustained response is underpinned by a distributed brain network of auditory cortical, frontal and hippocampal sources (Barascud et al., 2016; Southwell et al., 2017). Together, these sources are hypothesized to reflect the instantiation of a top-down model, producing increasingly reliable priors for upcoming sounds (Auksztulewicz et al., 2017).

In contrast, REG-RAND transitions evoke a mismatch response (similar in its dynamics to the MMN; Näätänen et al., 2007), followed by an abrupt drop in sustained activity, in line with immediate suppression of top-down prior expectations (see Barascud et al, 2016). The activity then settles at a low sustained level, consistent with the far weaker statistical constraints in the RAND pattern.

The PDR results point to a potential role for NE signaling in supporting the MEGindexed 'resetting response' observed during REG-RAND transitions. The relevant neural circuit may involve signaling from MMN-related brain systems (Auditory Cortex and right IFG; Garrido et al., 2008) to the LC-NE system, possibly via the ACC (Behrens et al., 2007; Karlsson et al., 2012; Payzan-LeNestour et al., 2013) or orbitofrontal cortex (Nogueira et al., 2017; 
Southwell and Chait, 2018). LC activation would then trigger NE-mediated rapid interruption of the temporo-frontal network associated with generating top-down prior expectations. Further investigation combining pupillometry and sensitive source imaging are necessary to identify these circuits, and to test the proposed linked between the MMN response and NE release.

Notably, the timing of the effects observed here implicate rapid signaling from the auditory system to the LC. The onset of the PDR observed here is at $\sim 500$ ms post transition; PDRs evoked by simple salient auditory or visual stimuli are commonly estimated to onset at 300ms (Hoeks and Levelt, 1993; Wang and Munoz, 2015), suggesting that the REG-RAND transition-related signal reaches the pupil no later than $\sim 200 \mathrm{~ms}$ post transition. This latency is within the same range as the abrupt drop in MEG activity recorded from auditory cortex (Fig. $1 B)$, thereby temporally aligning pupil dilation time with the putative signature of NE release.

\section{Substantial influence of attentional set on pupil responses to change in sequence structure}

Rendering the sequence transitions behaviorally relevant resulted in marked differences in pupil response dynamics. Notably, active monitoring of transitions gave rise to a PDR to $R A N D_{20}-R E G_{10}$ transitions and introduced a large delay $(300 \mathrm{~ms})$ to the PDR to the $R E G_{10}-R_{A N D}$ transitions. These effects were not strongly linked to the execution of a motor command, as evidenced by the fact that RT accounted for relatively little variance in various PDR metrics. Therefore, these behavior-related changes in the PDR to $R A N D_{20}-R E G_{10}$ and $R E G_{10}-R_{A N D}$ likely reflect a change in the functional state of the LC-NE system, or inputs to it. This may be a consequence of a behaviorally-driven emergence of a category boundary between REG and RAND, a richer representation of the statistics of the patterns before or after the transition, or a threshold change for model reset. That the STEP transition appears unaffected may be because this change can be detected relatively early in the auditory processing hierarchy, and thus may not depend on feedback information flow in the same way as do $R E G_{10}-R A N D_{20}$ and $R A N D_{20}-R_{E G}$. $_{10}$ 
We also observed a change in the dynamics of tonic pupil activity, i.e. in response to the ongoing sequence before the transition. When RAND and REG states were behaviorally irrelevant (in all but the third experiment), we observed no difference between the ongoing response to REG and RAND throughout the entire epoch (Fig. 2C,2D,4E,7B). However, making transitions between these states behaviorally relevant resulted in diverging PDRs to the different conditions themselves, even in the absence of a transition (Fig. 5B and 5D). Notably, these differences were observed even though the statistical structure per se is not explicitly trackable by listeners, due to the rapid rate at which successive tones are presented. Previous work has linked tonic pupil diameter differences to representation of expected uncertainty (Krishnamurthy et al., 2017; Nassar et al., 2012) possibly driven by cholinergic signaling (Reimer et al., 2016; Yu and Dayan, 2005). The present effects may be consistent with this interpretation; as indeed $R A N D_{20}$ is associated with less reliable priors than $R E G_{10}$. However, the fact that these differences in pupil diameter were observed exclusively during the active change detection task must therefore suggest that cholinergic activation is dependent on behavioral relevance and is not involved in automatic tracking of sequence predictability. An alternative, but not mutually exclusive, possibility is that this effect may reflect heightened vigilance or listening effort (Zekveld et al., 2014) arising through active sequence structure scanning, which is more demanding for RAND $_{20}$ (Southwell et al., 2017).

\section{Conclusions:}

The data reported here demonstrate that the pupil-linked LC-NE system plays an obligatory role in tracking and evaluating the statistics of unfolding sensory input, thereby supporting brain networks involved in maintaining flexible perceptual representations in changing environments. However, this system is confined in the circumstances under which it signals an interrupt, particularly in the absence of a remit from a task. Together with previous work in the decision-making and learning fields, the present results establish a unified view of NE as a model interrupt signal operating on multiple time scales, from those relevant to tracking 
reward environments in the context of decision making to tracking rapidly-unfolding sensory environments during perception.

\section{Acknowledgements}

We are grateful to Alex Billig and Shihab Shamma for comments and discussion and to Makoto Yoneya (NTT) for initiating the pupillometry setup at UCL. This work was supported by a BBSRC project grant and a BBSRC international partnering award to MC; SZ was partly funded by an NTT-UCL enhanced research contract. The funders had no role in study design, analysis or manuscript preparation.

\section{Author Contributions}

SZ conceived performed and analyzed the experiments; wrote manuscript.

MC supervised and administered the project; secured the funding; conceived the experiments; wrote the manuscript.

FD conceived the experiments; formal analysis of Experiment 3; wrote the manuscript.

PD provided expertise and feedback; wrote the manuscript.

SF provided expertise and feedback; commented on manuscript draft.

HL performed pilot experiments; provided expertise and feedback; commented on manuscript draft. 


\section{Declaration of Interests}

The authors declare no competing interests.

\section{References}

Aston-Jones, G., and Cohen, J.D. (2005). An Integrative Theory of Locus CoeruleusNorepinephrine Function: Adaptive Gain and Optimal Performance. Annu. Rev. Neurosci. 28, 403-450.

Aston-Jones, G., Rajkowski, J., and Kubiak, P. (1997). Conditioned responses of monkey locus coeruleus neurons anticipate acquisition of discriminative behavior in a vigilance task. Neuroscience 80, 697-715.

Auksztulewicz, R., Barascud, N., Cooray, G., Nobre, A.C., Chait, M., and Friston, K. (2017). The cumulative effects of predictability on synaptic gain in the auditory processing stream. J. Neurosci. 0291-17.

Avery, M.C., and Krichmar, J.L. (2017). Neuromodulatory Systems and Their Interactions: A Review of Models, Theories, and Experiments. Front. Neural Circuits 11.

Barascud, N., Pearce, M.T., Griffiths, T.D., Friston, K.J., and Chait, M. (2016). Brain responses in humans reveal ideal observer-like sensitivity to complex acoustic patterns. Proc. Natl. Acad. Sci. 201508523.

Bates, D., Mächler, M., Bolker, B., and Walker, S. (2014). Fitting Linear Mixed-Effects Models using Ime4. ArXiv14065823 Stat.

Behrens, T.E.J., Woolrich, M.W., Walton, M.E., and Rushworth, M.F.S. (2007). Learning the value of information in an uncertain world. Nat. Neurosci. 10, 1214-1221.

Bestmann, S., Harrison, L.M., Blankenburg, F., Mars, R.B., Haggard, P., Friston, K.J., and Rothwell, J.C. (2008). Influence of Uncertainty and Surprise on Human Corticospinal Excitability during Preparation for Action. Curr. Biol. 18, 775-780.

Bland, A.R., and Schaefer, A. (2012). Different Varieties of Uncertainty in Human DecisionMaking. Front. Neurosci. 6.

Bouret, S., and Sara, S.J. (2004). Reward expectation, orientation of attention and locus coeruleus-medial frontal cortex interplay during learning. Eur. J. Neurosci. 20, 791802. 
Bouret, S., and Sara, S.J. (2005). Network reset: a simplified overarching theory of locus coeruleus noradrenaline function. Trends Neurosci. 28, 574-582.

Brainard, D.H. (1997). The Psychophysics Toolbox. Spat. Vis. 10, 433-436.

Costa, V.D., and Rudebeck, P.H. (2016). More than Meets the Eye: the Relationship between Pupil Size and Locus Coeruleus Activity. Neuron 89, 8-10.

Dayan, P., and Abbott, L.F. (2001). Theoretical neuroscience (Cambridge, MA: MIT Press).

Dayan, P., and Yu, A.J. (2006). Phasic norepinephrine: A neural interrupt signal for unexpected events. Netw. Comput. Neural Syst. 17, 335-350.

Devauges, V., and Sara, S.J. (1990). Activation of the noradrenergic system facilitates an attentional shift in the rat. Behav. Brain Res. 39, 19-28.

Efron, B., and Tibshirani, R.J. (1994). An Introduction to the Bootstrap (CRC Press).

Einhäuser, W., Koch, C., and Carter, O.L. (2010). Pupil Dilation Betrays the Timing of Decisions. Front. Hum. Neurosci. 4.

Friston, K. (2005). A theory of cortical responses. Philos. Trans. R. Soc. B Biol. Sci. 360, 815836.

Garrido, M.I., Friston, K.J., Kiebel, S.J., Stephan, K.E., Baldeweg, T., and Kilner, J.M. (2008). The functional anatomy of the MMN: A DCM study of the roving paradigm. Neurolmage 42, 936-944.

Gershman, S.J., Jones, C.E., Norman, K.A., Monfils, M.-H., and Niv, Y. (2013). Gradual extinction prevents the return of fear: implications for the discovery of state. Front. Behav. Neurosci. 7.

Hoeks, B., and Levelt, W.J.M. (1993). Pupillary dilation as a measure of attention: a quantitative system analysis. Behav. Res. Methods Instrum. Comput. 25, 16-26.

Horváth, J., and Winkler, I. (2004). How the human auditory system treats repetition amongst change. Neurosci. Lett. 368, 157-161.

Howlett, J.R., Huang, H., Hysek, C.M., and Paulus, M.P. (2017). The effect of single-dose methylphenidate on the rate of error-driven learning in healthy males: a randomized controlled trial. Psychopharmacology (Berl.) 234, 3353-3360.

Jaeger, B.C., Edwards, L.J., Das, K., and Sen, P.K. (2017). An R2 statistic for fixed effects in the generalized linear mixed model. J. Appl. Stat. 44, 1086-1105.

Jepma, M., and Nieuwenhuis, S. (2011). Pupil Diameter Predicts Changes in the ExplorationExploitation Trade-off: Evidence for the Adaptive Gain Theory. J. Cogn. Neurosci. 23, 1587-1596.

Joshi, S., Li, Y., Kalwani, R.M., and Gold, J.I. (2016). Relationships between Pupil Diameter and Neuronal Activity in the Locus Coeruleus, Colliculi, and Cingulate Cortex. Neuron $89,221-234$. 
Karlsson, M.P., Tervo, D.G.R., and Karpova, A.Y. (2012). Network Resets in Medial Prefrontal Cortex Mark the Onset of Behavioral Uncertainty. Science 338, 135-139.

Knill, D.C., and Richards, W. (1996). Perception as Bayesian inference (Cambridge University Press).

Krishnamurthy, K., Nassar, M.R., Sarode, S., and Gold, J.I. (2017). Arousal-related adjustments of perceptual biases optimize perception in dynamic environments. Nat. Hum. Behav. 1, s41562-017-0107-017.

Lavín, C., San Martín, R., and Rosales Jubal, E. (2014). Pupil dilation signals uncertainty and surprise in a learning gambling task. Front. Behav. Neurosci. 7.

Liao, H.-I., Yoneya, M., Kidani, S., Kashino, M., and Furukawa, S. (2016). Human Pupillary Dilation Response to Deviant Auditory Stimuli: Effects of Stimulus Properties and Voluntary Attention. Front. Neurosci. 10.

Maris, E., and Oostenveld, R. (2007). Nonparametric statistical testing of EEG- and MEG-data. J. Neurosci. Methods 164, 177-190.

Marshall, L., Mathys, C., Ruge, D., Berker, A.O. de, Dayan, P., Stephan, K.E., and Bestmann, S. (2016). Pharmacological Fingerprints of Contextual Uncertainty. PLOS Biol. 14, e1002575.

McGaughy, J., Ross, R.S., and Eichenbaum, H. (2008). Noradrenergic, but not cholinergic, deafferentation of prefrontal cortex impairs attentional set-shifting. Neuroscience 153, 63-71.

Näätänen, R., Paavilainen, P., Rinne, T., and Alho, K. (2007). The mismatch negativity (MMN) in basic research of central auditory processing: A review. Clin. Neurophysiol. 118, 2544-2590.

Nakagawa, S., and Schielzeth, H. (2013). A general and simple method for obtaining R2 from generalized linear mixed-effects models. Methods Ecol. Evol. 4, 133-142.

Nassar, M.R., Rumsey, K.M., Wilson, R.C., Parikh, K., Heasly, B., and Gold, J.I. (2012). Rational regulation of learning dynamics by pupil-linked arousal systems. Nat. Neurosci. 15, 1040-1046.

Nieuwenhuis, S., De Geus, E.J., and Aston-Jones, G. (2011). The anatomical and functional relationship between the P3 and autonomic components of the orienting response: P3 and orienting response. Psychophysiology 48, 162-175.

Nogueira, R., Abolafia, J.M., Drugowitsch, J., Balaguer-Ballester, E., Sanchez-Vives, M.V., and Moreno-Bote, R. (2017). Lateral orbitofrontal cortex anticipates choices and integrates prior with current information. Nat. Commun. 8, 14823.

Oostenveld, R., Fries, P., Maris, E., Schoffelen, J.-M., Oostenveld, R., Fries, P., Maris, E., and Schoffelen, J.-M. (2010). FieldTrip: Open Source Software for Advanced Analysis of MEG, EEG, and Invasive Electrophysiological Data, FieldTrip: Open Source Software for Advanced Analysis of MEG, EEG, and Invasive Electrophysiological Data. Comput. Intell. Neurosci. Comput. Intell. Neurosci. 2011, 2011, e156869. 
O'Reilly, J.X. (2013). Making predictions in a changing world-inference, uncertainty, and learning. Front. Neurosci. 7.

Payzan-LeNestour, E., Dunne, S., Bossaerts, P., and O'Doherty, J.P. (2013). The Neural Representation of Unexpected Uncertainty during Value-Based Decision Making. Neuron 79, 191-201.

Preuschoff, K., 't Hart, B.M., and Einhäuser, W. (2011). Pupil Dilation Signals Surprise: Evidence for Noradrenaline's Role in Decision Making. Front. Neurosci. 5.

Rao, R.P.N. (2005). Bayesian inference and attentional modulation in the visual cortex. Neuroreport 16, 1843-1848.

Reimer, J., McGinley, M.J., Liu, Y., Rodenkirch, C., Wang, Q., McCormick, D.A., and Tolias, A.S. (2016). Pupil fluctuations track rapid changes in adrenergic and cholinergic activity in cortex. Nat. Commun. 7, 13289.

Rolfs, M., Kliegl, R., and Engbert, R. (2008). Toward a model of microsaccade generation: The case of microsaccadic inhibition. J. Vis. 8, 5-5.

Rosburg, T. (2004). Effects of tone repetition on auditory evoked neuromagnetic fields. Clin. Neurophysiol. 115, 898-905.

Rushworth, M.F.S., and Behrens, T.E.J. (2008). Choice, uncertainty and value in prefrontal and cingulate cortex. Nat. Neurosci. 11, 389-397.

Samuels, E.R., and Szabadi, E. (2008a). Functional Neuroanatomy of the Noradrenergic Locus Coeruleus: Its Roles in the Regulation of Arousal and Autonomic Function Part I: Principles of Functional Organisation. Curr. Neuropharmacol. 6, 235-253.

Samuels, E.R., and Szabadi, E. (2008b). Functional Neuroanatomy of the Noradrenergic Locus Coeruleus: Its Roles in the Regulation of Arousal and Autonomic Function Part II: Physiological and Pharmacological Manipulations and Pathological Alterations of Locus Coeruleus Activity in Humans. Curr. Neuropharmacol. 6, 254-285.

Sara, S.J., and Bouret, S. (2012). Orienting and Reorienting: The Locus Coeruleus Mediates Cognition through Arousal. Neuron 76, 130-141.

Sara, S.J., and Segal, M. (1991). Chapter 40 - Plasticity of sensory responses of locus coeruleus neurons in the behaving rat: implications for cognition. In Progress in Brain Research, C.D. Barnes, and O. Pompeiano, eds. (Elsevier), pp. 571-585.

Sohoglu, E., and Chait, M. (2016). Detecting and representing predictable structure during auditory scene analysis. ELife 5, e19113.

Southwell, R., and Chait, M. (2018). Enhanced deviant responses in patterned relative to random sound sequences. Cortex 109, 92-103.

Southwell, R., Baumann, A., Gal, C., Barascud, N., Friston, K., and Chait, M. (2017). Is predictability salient? A study of attentional capture by auditory patterns. Phil Trans $R$ Soc B 372, 20160105. 
Turk-Browne, N.B., Scholl, B.J., Johnson, M.K., and Chun, M.M. (2010). Implicit perceptual anticipation triggered by statistical learning. J. Neurosci. Off. J. Soc. Neurosci. 30, 11177-11187.

Vankov, A., Hervé-Minvielle, A., and Sara, S.J. (1995). Response to Novelty and its Rapid Habituation in Locus Coeruleus Neurons of the Freely Exploring Rat. Eur. J. Neurosci. 7, 1180-1187.

Wang, C.-A., and Munoz, D.P. (2015). A circuit for pupil orienting responses: implications for cognitive modulation of pupil size. Curr. Opin. Neurobiol. 33, 134-140.

Warren, R.M. (2008). Auditory Perception: An Analysis and Synthesis (Cambridge: Cambridge University Press).

Widmann, A., Engbert, R., and Schröger, E. (2014). Microsaccadic responses indicate fast categorization of sounds: a novel approach to study auditory cognition. J. Neurosci. Off. J. Soc. Neurosci. 34, 11152-11158.

Wolff, C., and Schröger, E. (2001). Activation of the auditory pre-attentive change detection system by tone repetitions with fast stimulation rate. Cogn. Brain Res. 10, 323-327.

Yu, A.J., and Dayan, P. (2005). Uncertainty, Neuromodulation, and Attention. Neuron 46, 681692.

Zekveld, A.A., Heslenfeld, D.J., Johnsrude, I.S., Versfeld, N.J., and Kramer, S.E. (2014). The eye as a window to the listening brain: Neural correlates of pupil size as a measure of cognitive listening load. Neurolmage 101, 76-86.

\section{Methods}

\section{CONTACT FOR REAGENT AND RESOURCE SHARING}

Further information and requests for resources and data should be directed to and will be fulfilled by the Lead Contact: Maria Chait (m.chait@ucl.ac.uk)

\section{SUBJECT DETAILS}

Ethics declaration: The experimental procedures were approved by the Research Ethics Committee of University College London. Participants were provided written informed consent and were paid for their participation. 
Participant exclusion criteria and justification of $\mathbf{N}$ : The following exclusionary criteria were consistently applied across all experiments: To ensure that observed changes in pupil diameter were not blink-related artifacts, participants were excluded if they blinked in more than $50 \%$ of trials. Additionally, participants were excluded if their mean gaze location exceeded three standard deviations from the group mean.

The experiments were not conducted in the order in which they are reported. Initial experiments involved larger numbers $(\mathrm{N}=20)$. Over time, and as the first author became more experienced with conducting the experiments, it was concluded that smaller $\mathrm{N}$ and fewer trials per condition were sufficient.

\section{Participant details:}

All participants reported normal hearing, normal or corrected-to-normal vision, and no history of neurological disorders.

Experiment 1A: Data from 18 participants (11 females; aged 20-29, average 23.41) are presented. Data from one additional participant were excluded due to failure to complete the experiment. Two further participants were excluded due to high blink rates in the STEP condition.

Experiment 1B: Data from 14 new participants (13 females; aged 22-26, average 23.1) were used in the analysis. Five additional participants were excluded due to high blinks rates. One further participant was excluded due to poor behavioral performance $(0 \%$ gap detection hit rate in REG sequences).

Experiment 2: Data from 18 new participants (15 females; aged 20-35, average 25.1) are reported. Two additional participants were excluded: one due to high blink rates, and one due to wandering gaze.

Experiment 3: Data from 14 participants (10 females; aged 22-30, average 24.3) are presented. None were excluded. 
Experiment 4A: Data from 12 new participants (9 females; aged 21 - 26, average 23.6) are presented. None were excluded.

Experiment 4B: This experiment was performed twice; a total of 30 new participants took part, with 15 participants initially (11 females; aged 20-29, average 23.5) and a new group of 15 participants subsequently (14 females; aged 20-25, average 22.5 ) to replicate the results of the first cohort. None were excluded.

\section{METHOD DETAILS}

\section{Pupil size measurement and analysis}

Participants sat in front of a monitor at a viewing distance of $60 \mathrm{~cm}$ in a dimly lit, acoustically shielded room (IAC triple walled sound-attenuating booth), with their head supported on a chinrest. They were instructed to continuously fixate at a white cross presented at the center of the screen (BENQ XL2420T with resolution of 1920x1080; refresh rate of $60 \mathrm{~Hz}$ ) against a black background. The visual display remained constant throughout the session. An infrared eye-tracking camera (Eyelink 1000 Desktop Mount, SR Research Ltd.), positioned just below the monitor, continuously tracked gaze position and recorded pupil diameters, focusing binocularly at a sampling rate of $1000 \mathrm{~Hz}$. The standard five-point calibration procedure for the Eyelink system was conducted prior to each experimental block. Participants were instructed to blink naturally.

Only the left eye was analyzed. To avoid contamination by blinks, which tended to increase towards the end of the stimulus, the final $0.5 \mathrm{~s}$ of each trial were cut from the analysis. The epochs therefore spanned one second before to two seconds post transition (Experiment 1) or three seconds post transition (all other experiments). This cut-off was comfortably beyond the time needed to detect the transitions, as corroborated by behavioral and MEG results (Experiment 3 and Barascud et al., 2016). Data in each epoch were smoothed with a 150ms Hanning window, z-scored for each block and baseline-corrected by subtracting the median 
pupil size of the pre-transition baseline. Intervals where the eye tracker detected full or partial eye closure were automatically treated as missing data and recovered with shape-preserving piecewise cubic interpolation. Trials with more than $50 \%$ missing data were excluded from analysis (<2 trials per subject). The normalized pupil diameter was time-domain-averaged across all epochs of each condition type to produce a single time series for each condition. Matched no-transition conditions were epoched in a similar manner around 'dummy' transition times set to match those in the transition conditions. To compare pupil dynamics from sequence onset, the data in the no change conditions (REG, RAND, CONST) were epoched from 1 second before sequence onset to 6 seconds post-onset and processed as described above.

\section{Pupil event rate analysis}

Pupil event rate analysis compared the incidence of pupil dilation or constriction events. Following Joshi et al, (2016), events were defined as local minima (dilations; PD) or local maxima (constrictions; PC) with the constraint that continuous dilation or constriction is maintained for at least 75ms (yellow dots in Figure 3) or 300ms (black dots in Figure 3). Both thresholds provided consistent data (see results), as did intermediate thresholds (including $100 \mathrm{~ms}$ and $125 \mathrm{~ms}$; not shown). The rate was estimated for each subject separately by using a sliding 500ms window over all trials in each condition and comparing rate changes across time and condition (see 'Statistical Analysis', below). This relatively long window enabled us to capture possible subtle changes in the rate of occurrence of PD events. The analysis interval was between 2 seconds before to two seconds after the transition. Previous work (Barascud et al., 2016) demonstrated that brain responses to the transitions occur within $<300 \mathrm{~ms}$ and behavioral responses (button press) are completed by 1000ms (see also Exp. 3 below), thus suggesting that the analysis interval is appropriate for revealing any effects. We also estimated rate by tallying PD or PC events with non-overlapping 500ms windows, and by convolving with an impulse function (see also Joshi et al., 2016; Rolfs et al., 2008). For each condition, in each 
participant and trial, the event time series were summed and normalized by the number of trials and the sampling rate. Then, a causal smoothing kernel $\omega(\tau)=\alpha^{2} \times \tau \times e^{-\alpha \tau}$ was applied with a decay parameter of $\alpha=\frac{1}{50} \mathrm{~ms}$ (Dayan and Abbott, 2001; Rolfs et al., 2008; Widmann et al., 2014). All analyses yielded identical results, therefore only the former is reported.

\section{Experiment 1A Stimuli and procedure}

The stimuli were sequences of concatenated tone-pips $(50 \mathrm{~ms})$ with frequencies drawn from a pool of 20 fixed values (log-spaced) between 200 and $2000 \mathrm{~Hz}$. The tone-pips were arranged according to six frequency patterns, generated anew for each participant (Figure 1): CONST sequences consisted of a single repeating tone, chosen by randomly selecting a frequency from the pool on each trial; STEP sequences consisted of a step change from one repeating tone to another repeating tone of a different frequency (both frequencies randomly drawn on each trial); $\mathbf{R E G}_{10}$ sequences were generated by randomly selecting (with replacement) 10 frequencies from the pool and then iterating that sequence to create a regularly repeating pattern (with new patterns generated on each trial); $\mathbf{R A N D}_{20}$ sequences were generated by randomly sampling frequencies from the pool with replacement; $\mathbf{R E G}_{10^{-}} \mathbf{R A N D}_{20}$ and $\mathbf{R A N D}_{\mathbf{2 0}}$ $\mathbf{R E G}_{10}$ sequences contained a transition between a regular pattern and a random pattern. The stimulus length varied randomly between 5 and 7 seconds, with a jittered transition time at around 2.5- and 3.5-seconds post-onset.

Sounds were presented diotically through headphones (Sennheiser HD558) via a Creative Sound Blaster X-Fi sound card (Creative Technology, Ltd.) at a comfortable listening level, self-adjusted by each participant. Stimulus presentation and response recording were controlled with the Psychtoolbox package (Psychophysics Toolbox Version 3; Brainard, 1997) in MATLAB (The MathWorks, Inc.). 146 stimuli - 24 for each condition - were randomly presented in four consecutive blocks (separated by 3 min breaks) with an inter-trial interval of 
six seconds. $25 \%$ of the signals contained a silent gap, occurring at any time from $250 \mathrm{~ms}$ post onset to $750 \mathrm{~ms}$ pre-offset. Participants were instructed to monitor the sequences for these events and to respond by pressing a button as quickly as possible. To equate for task difficulty, the gap consisted of one missing tone $(50 \mathrm{~ms})$ in the CONST and STEP sequences, and two missing tones (100ms) in REG and RAND sequences. Visual feedback, lasting $400 \mathrm{ms,} \mathrm{was}$ provided immediately at the end of each sequence. Trials containing a gap and trials in which participants made a false positive were excluded from further analysis.

\section{Experiment 1B Stimuli and Procedure}

The stimuli and procedure were identical to Experiment $1 \mathrm{~A}$, except that only three blocks were run, for a total of 108 stimuli. The data from Experiment $1 \mathrm{~A}$ indicated that this was sufficient to measure the relevant effects. To address behavioral differences between conditions observed in Experiment 1A, the gap in RAND sequences was lengthened to 3 tones (150ms).

\section{Experiment 2 Stimuli and Procedure}

The stimulus set is described in Figure 4. Stimulus length was randomly varied between 6-8 seconds, with the transition jittered between 3-4 seconds after sequence onset. A total of 240 stimuli were presented in random order over 5 consecutive blocks: 60 REG ${ }_{10}, 20$ REG ${ }_{10^{-}}$ RAND $_{10}, 20$ REG $_{10}-$ RAND $_{10 d}, 20$ REG $_{10}-R$ RND $_{20}, 40$ RAND $_{10}, 20$ RAND $_{10}-R^{-} G_{10}, 20$ RAND $_{10}-$ $R E G_{10 d}, 20 R A N D_{20}$ and $20 R A N D_{20}-R G_{10} .20 \%$ of the sequences contained a gap, with equal probability spread across conditions. Gap lengths were as in Experiment 1B.

\section{Experiment 3 Stimuli and Procedure}

The stimulus set was identical to that in Experiment 1 but that stimuli contained no gaps. Participants were instructed to press a button as quickly as possible after detecting a pattern change in the sound sequence. Key presses were checked after each tone, so the resolution of the reaction time measurement was $50 \mathrm{~ms}$. In total 150 stimuli were presented in 
random order over 5 consecutive blocks - 25 of each condition -with an inter-trial interval of five to seven seconds.

\section{Experiment 4A Stimuli and Procedure}

The stimulus set consisted of the conditions used in Experiment 1 (Figure 1) and additionally included a new condition: $\mathbf{R A N D _ { 2 0 } - \mathbf { R E G }}$ - which consisted of a transition from a random sequence to a sequence of fixed frequency tones $\left(R E G_{1}\right)$ (see Figure 7). In total, 288 trials were presented in random order over 8 consecutive blocks: $24 R_{A N D} 0^{-} R E G_{1}, 24 R A N D_{20}{ }^{-}$ $\mathrm{REG}_{10}, 48 \mathrm{RAND}_{20}, 48 \mathrm{REG} \mathrm{G}_{10}, 48 \mathrm{REG} \mathrm{G}_{10}-\mathrm{RAND}_{20}, 48$ CONT and 48 STEP, with one-third of the sequences containing a gap (lengths as in Experiment 1B).

\section{Experiment 4B Stimuli and Procedure}

The stimulus set was expanded to include two additional conditions: $\mathbf{R A N D}_{\mathbf{2 0}} \mathbf{-} \mathbf{R E G _ { 2 }}$ consisted of a transition from a random sequence to a sequence to a regular pattern of two alternating tones; $\mathbf{R A N D}_{20}-\mathbf{R E G}_{5}$ consisted of a transition from a random sequence to a sequence to a regular pattern of five alternating tones (Figure 7). Overall, 168 stimuli were presented in random order over 7 consecutive blocks including $21 \mathrm{RAND}_{20}-\mathrm{REG}_{1}, 21 \mathrm{RAND}_{20^{-}}$ $R E G_{2}, 21 R A N D_{20}-R E G_{5}, 21 R A N D_{20}-R^{2} G_{10}$ and $84 R A N D_{20}$, with one-third of the sequences containing a gap.

\section{QUANTIFICATION AND STATISTICAL ANALYSIS}

Comparison of PDRs across conditions: A series of paired t-tests were conducted on each pair of conditions (two-tailed; over the entire epoch length; downsampled to $20 \mathrm{~Hz}$ ), with familywise error (FWE) control using a non-parametric permutation procedure with 5,000 iterations (cluster-defining height threshold of $p<0.05$ with an FWE-corrected cluster size threshold of $p$ $<0.05$; Maris and Oostenveld, 2007), as implemented in the Fieldtrip toolbox 
(http://www.fieldtriptoolbox.org; Oostenveld et al., 2010). Significant time intervals are presented as colored horizontal bars below the PDR plots.

Event rate analysis: Because PD/PC events are rare (normal pre-transition rates are 1 2 per second) the statistical analysis was conducted by pooling over Experiment $1 \mathrm{~A}$ and $\mathrm{B}$ (32 subjects overall). The cluster analysis used to compare PDR was conducted between each transition condition and its control with other details as described above.

Experiment 3: To quantify the change in PDR peak latency for STEP and REG ${ }_{10}-R_{A N D} D_{20}$ in Experiment 3 (active transition detection) relative to Experiments 1,2,4,5 (gap detection), bootstrap analysis (1000 iterations; Efron and Tibshirani, 1994) was performed on two sets of participant data, one constructed from the 14 active participants in Experiment 3 ('active') and another from the 57 participants pooled from Experiments 1,2,4,5 ('non-active'). On each iteration, a 'simulated' PDR latency was computed over 14 participants randomly drawn from the 'non-active' pool. The scatterplots in Figure 5C show the distribution of the simulated peak latency of STEP (left) and $R E G_{10}-R_{A N D}$ (right) in the 'non-active' pool. The red crosses indicate the mean peak latency in Experiment 3.

We analyzed potential single-trial level associations between reaction time and pupil responses using REML in JMP 13.2 (SAS Institute, SAS Institute Inc., Cary, NC). Because reaction times (RTs) and pupillometry measures were non-normally distributed, rank scores were used for all analyses (ties assigned the bottom rank from the set of same values). Three measures of PDR dynamics were investigated: 'Max PDR amplitude', reflected the peak pupil diameter; 'Max PDR latency', reflected the latency of the PDR peak, 'max derivative PDR latency' measured the time of maximum pupil rate of change. Participant was entered as the random factor, and rank RT as fixed effect; for reported analyses, slope was fixed over subjects to avoid potential overfitting, but all effects at $p<0.05$ also hold when separate slopes are fit for each participant (except when noted). We report t- and p-values associated with the RT fixed effect; we also provide an estimate of relative contribution of the fixed effect to overall 
bioRxiv preprint doi: https://doi.org/10.1101/466367; this version posted November 8, 2018. The copyright holder for this preprint (which was not certified by peer review) is the author/funder, who has granted bioRxiv a license to display the preprint in perpetuity. It is made available under aCC-BY-NC-ND 4.0 International license.

model fit by computing partial $\mathrm{R}^{2}$ estimates using the Ime4 and $\mathrm{r} 2 \mathrm{glmm}$ (Jaeger et al., 2017) packages in R; these provide an implementation of the Nakagawa and Schielzeth (2013) algorithm.

\section{DATA AVAILABILITY}

Data will be made freely available to readers from the date of publication. Specific details of the relevant repository will be provided upon manuscript acceptance. 


\section{Supplementary Materials}

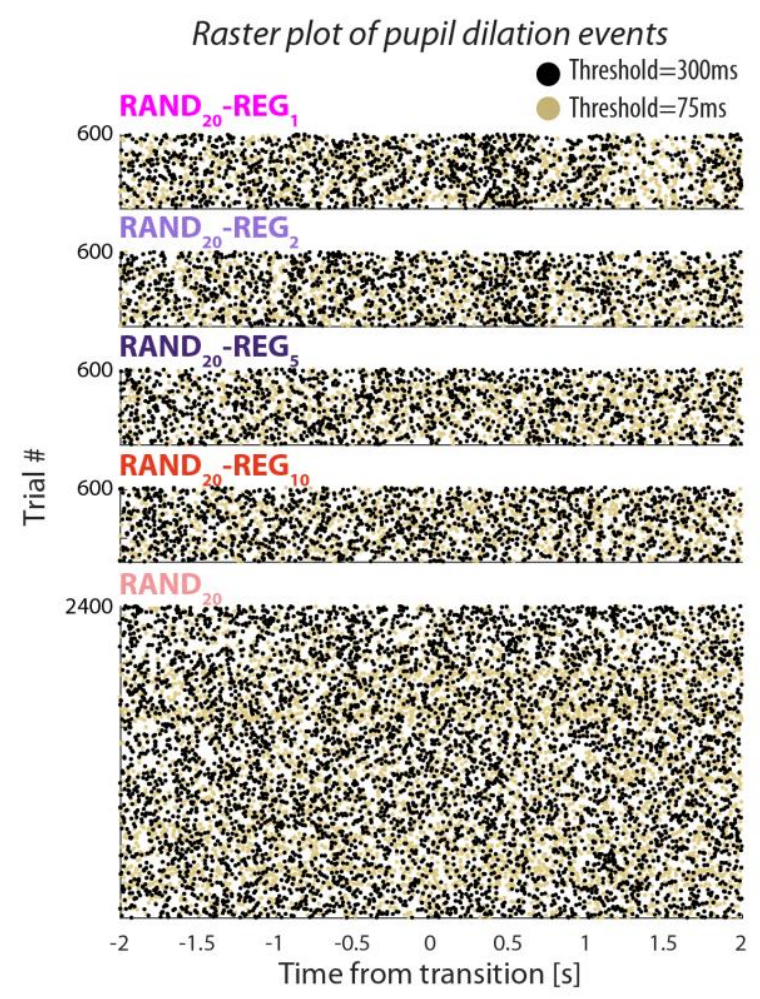

Raster plot of pupil constriction events

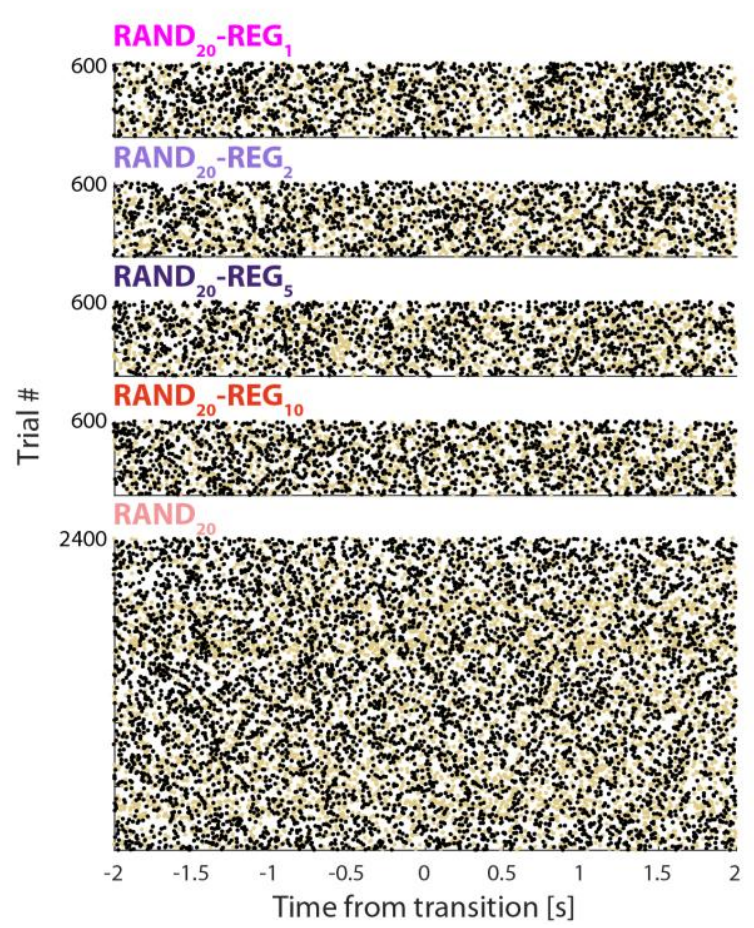

Pupil dilation rate
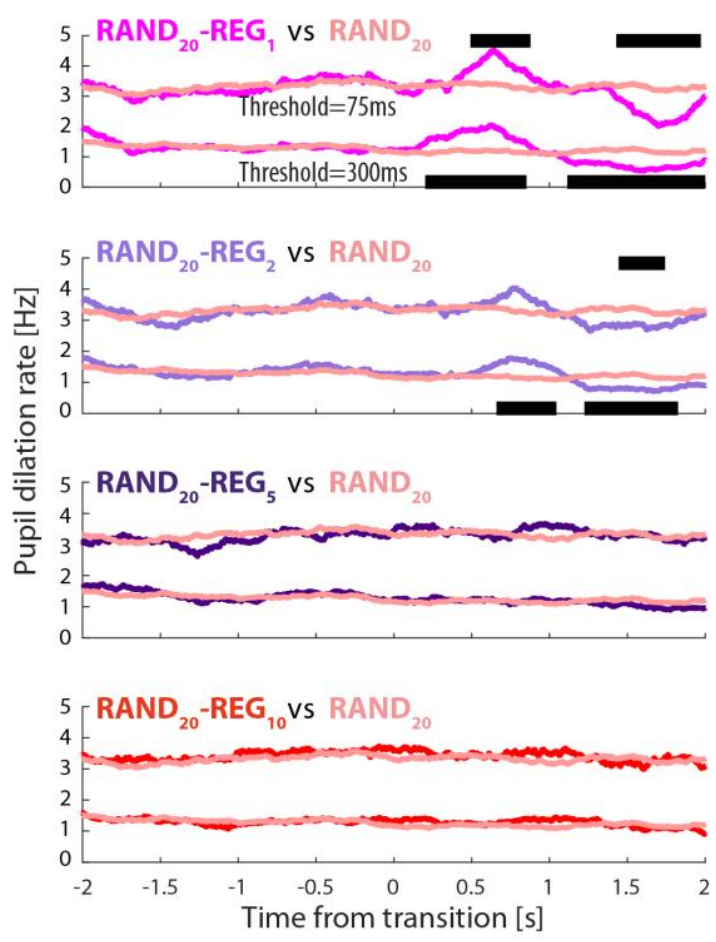

Pupil constriction rate
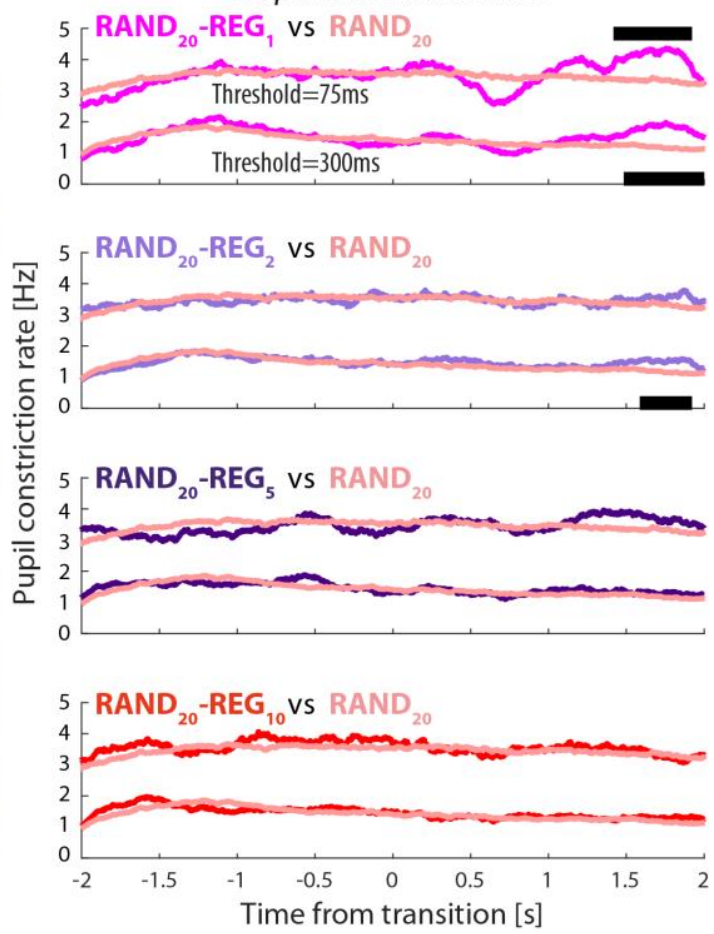
Figure S1: Experiment 4B: Pupil dilation and constriction rates. [Top left] Raster plots of pupil dilation (PD) events extracted from all trials and all participants (collapsed over the two groups, $N=30$ ). Each line represents a single trial. Black dots represent the onset of a pupil dilation with a duration of at least $300 \mathrm{~ms}$, yellow dots represent pupil dilation onsets with a threshold duration of $75 \mathrm{~ms}$. Transition time is indicated by a black vertical line. [Top right] Pupil dilation rate (running average with a 500ms window) as a function of time relative to the transition. From top to bottom, one of four transition conditions are plotted against the no-change control RAND ${ }_{20}:$ RAND $_{20}-R^{2} G_{1}, R^{2} N_{20}=$ $R E G_{2}, R A N D_{20}-R E G_{5}$ and $R A N D_{20}-R E_{10}$. The black horizontal lines indicate time intervals where cluster-level statistics showed significant differences between each change condition and the no-change control RAND 20 . The statistics for the PD rates with a threshold duration of $75 \mathrm{~ms}$ and $300 \mathrm{~ms}$ are placed above and below the graph, respectively. The lower panels present the pupil constriction (PC) rate results, arranged in the same format. 